\title{
Microvibrations Induced by a Cantilevered Wheel Assembly with a Soft-Suspension System
}

\author{
Zhe Zhang* and Guglielmo S. Aglietti \pm \\ University of Southampton, Southampton, Hampshire, England SO17 1BJ, United Kingdom \\ and \\ Weiyong Zhou \\ National University of Defense Technology, 410073 Hunan, People's Republic of China
}

DOI: $\underline{10.2514 / 1 . J 050791}$

\begin{abstract}
Microvibration management onboard spacecraft with high stability requirements has drawn increasing interest from engineers and scientists, and this paper discusses a reaction wheel design that allows a significant reduction of mid- to high-frequency microvibrations and that has been practically implemented in industry. Disturbances typically induced by mechanical systems onboard a spacecraft (especially rotating devices such as reaction wheel assemblies and momentum wheel assemblies) can severely degrade the performance of sensitive instruments. Traditionally, wheel-induced high-frequency (over 100-200 Hz) vibrations, generated by a combination of phenomena from bearing noise to dynamic amplifications due to internal resonances, are especially difficult to control. In this paper, the dynamic behavior of a newly designed wheel assembly, with a cantilevered flywheel configuration supported by a soft-suspension system, is investigated. The wheel assembly's mathematical model is developed and later verified with vibration tests. Wheel-assembly-induced lateral and axial microvibrations are accurately measured using a seismic-mass microvibration measurement system, which represents an alternative to typical microvibration measurement setups. Finally, the performance of this wheel assembly in terms of microvibration emissions is compared with a traditional design (with a rigid suspension) through comparison of frequency spectra, and it is shown that this design produces significantly lower vibrations at high frequency.
\end{abstract}

$\begin{array}{lll}a & = & \text { acceleration } \\ C & = & \text { center of mass of the seismic mass } \\ c & = & \text { ashpot damping coefficient } \\ d & = & \text { shaft length } \\ d_{1}, d_{2} & = & \text { vertical distance of accelerometers } 1 \text { and } 2 \\ & & \text { to the system center of mass } \\ d_{3} & = & \text { seismic-mass half-width } \\ \boldsymbol{F} & = & \text { external excitation vector } \\ F & = & \text { force } \\ \boldsymbol{G} & = & \text { gyroscopic matrix } \\ h & = & \text { flywheel disc half-height } \\ \boldsymbol{I} & = & \text { inertia tensor } \\ I & = & \text { moment of inertia } \\ I_{r}, I_{z} & = & \text { balanced flywheel transverse and polar } \\ \boldsymbol{K} & = & \text { moment of inertia } \\ k & = & \text { stiffness matrix } \\ L & = & \text { Lagrangian } \\ l & = & \text { vertical distance from the soft-suspension-system } \\ & = & \text { interface to the seismic-mass center of mass } \\ M & = & \text { mass matrix } \\ \boldsymbol{M} & = & \text { dynamic and static mass imbalance } \\ m_{d}, m_{s} & = & \text { extra point mass attached on flywheel } \\ m_{e} & \end{array}$

Received 10 August 2010; revision received 17 December 2010; accepted for publication 19 December 2010. Copyright (C) 2010 by Zhe Zhang. Published by the American Institute of Aeronautics and Astronautics, Inc., with permission. Copies of this paper may be made for personal or internal use, on condition that the copier pay the $\$ 10.00$ per-copy fee to the Copyright Clearance Center, Inc., 222 Rosewood Drive, Danvers, MA 01923; include the code 0001-1452/11 and $\$ 10.00$ in correspondence with the CCC.

${ }^{*}$ Graduate Student, Astronautics Research Group, School of Engineering Sciences; zz1e08@soton.ac.uk.

${ }^{\dagger}$ Head of Group, Astronautics Research Group, School of Engineering Sciences; gsa@soton.ac.uk.

Graduate Student, Aerospace and Material Engineering, Changsha; wz1c09@soton.ac.uk.

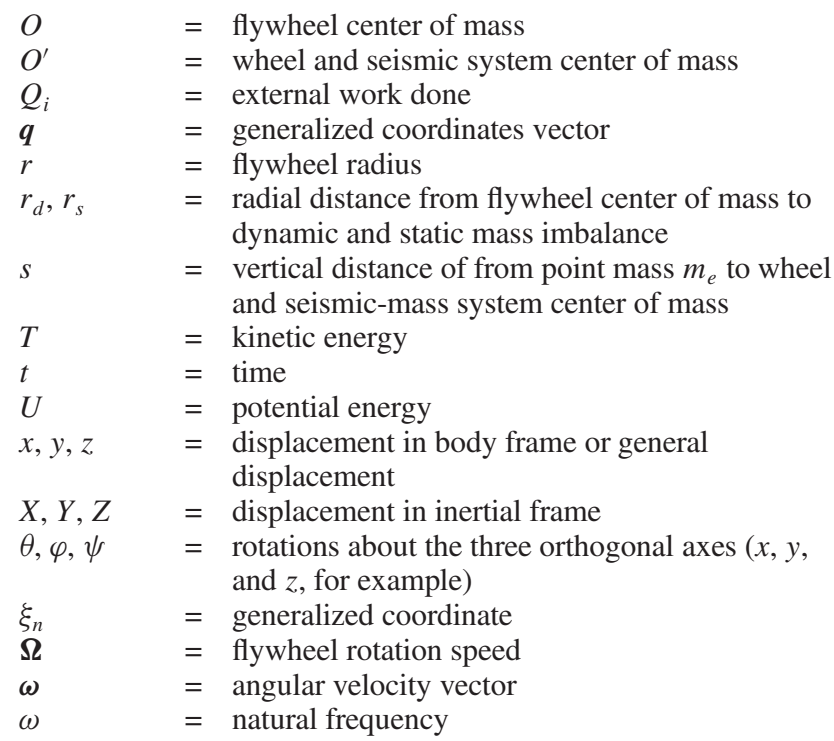

\section{Subscripts}

$c \quad=$ seismic mass

$c_{x x}, c_{y y}=$ about $x_{c}$ and $y_{c}$ axis of seismic mass

$i x, i y=$ at wheel-assembly/seismic-mass interface, in $x$ and $y$ directions

md $\quad=$ dynamic imbalance condition

$m s \quad=$ static imbalance condition

$r \quad=$ torsional (spring and dashpot)

$s \quad=$ wheel and seismic system

$s_{x x}, s_{y y}, s_{z z}=$ orthogonal directions of wheel-assembly and seismic-mass system

$s x, s y, s z \quad=\quad$ orthogonal directions of wheel-assembly and seismic-mass system

$t \quad=$ linear (spring and dashpot)

$w \quad=$ flywheel

$x, y, z \quad=$ orthogonal directions 


\section{Introduction}

$\mathbf{M}$ INIMIZING microvibrations onboard spacecraft is a key issue for current and future space systems that require high pointing accuracy and stringent stability performance [1 $1-3]$, for example. In this context, the term microvibration refers to low-level mechanical disturbances in the region of microgravity, typically occurring at frequencies from less than $1 \mathrm{~Hz}$ up to $1 \mathrm{kHz}$ [4]. Microvibrations are mainly generated by mechanical systems located on the spacecraft, including cryocoolers, solar array drive mechanisms, drives for pointing mechanisms, and rotating devices such as reaction wheel assemblies (RWAs) and momentum wheel assemblies (MWAs), collectively referred to here as wheel assemblies (WAs). Among all devices onboard a spacecraft, WAs are usually the largest microvibration sources []] , vibration forces and moments emitted by WAs can severely degrade the performance of precision payloads in space [6-10]. Therefore, understanding and controlling their vibration (either passively or actively, for example, [11-13]) is a crucial factor to achieving the desired level of payload performance.

A typical WA consists of a rotating flywheel mounted on a rigid shaft supported by bearings (mechanical or magnetic) and driven by a brushless dc motor, all of which is encased in a housing. Nominally, RWAs have zero speed and may be rotated (accelerated/decelerated) in either direction, generally up to $3000-4000 \mathrm{rpm}$, to produce reactive torques used to control the attitude of the satellite. MWAs usually spin at a high mean speed (typically between 5000 and $10,000 \mathrm{rpm}$ ) to provide momentum bias and stability to the spacecraft [14]. Both wheel types are often used in conjunction with external torquers and are particularly useful when the spacecraft needs to be rotated by very small amounts and to maintain pointing without consuming onboard propellant [15].

Common flywheel configurations of a WA are either symmetrical (flywheel at midspan of the shaft) or cantilevered (flywheel at one end of the shaft). Though the configurations are different, as a rotating mechanical system they manifest similar dynamic behavior, except that the two flexural modes (lateral rocking and translational) are mixed together for the cantilevered type, but well defined and separate for the symmetrical type (this is true especially for WAs in the case that the shaft is short and rigid $[16,17])$. Although rotor dynamics has been studied for many years, the first detailed dynamic analysis of a symmetrical flywheel for a space application was proposed in $[18,19]$ for the RWAs used in Hubble Space Telescope in the 1990s. The wheel-imbalance model with flywheel static imbalance [offset of the center of mass (c.m.) of the flywheel with respect to its spin axis] and dynamic imbalance (misalignment of the flywheel's principal axis and the rotation axis) was conceptualized, and other disturbance sources (bearing and motor) impact on a typical RWA were analyzed in [20]. RWA testing in [18] showed that the induced disturbances are mainly generated by four phenomena: flywheel imbalance, flywheel internal flexibility, bearing disturbance, and motor disturbance. Among these, the flywheel imbalance is generally regarded as the largest. The initial test results from [19] showed (not surprisingly, as this is typical for general rotating machinery) that the wheel-induced vibrations are mostly sinusoidal (often multitone) in nature, and that below the first resonant frequency, imbalance causes a disturbance force and moment, respectively, at the flywheel's spin rate (the fundamental harmonic) with amplitude proportional to the rotational speed squared. However, a detailed model of the RWA-induced disturbances was not published until 1999 in $[21,22]$.

Based on the RWA vibration tests carried out at NASA Goddard Space Flight Center (GSFC) and Orbital Sciences Corporation in [21], an empirical model of a symmetrical RWA was developed to extract harmonic parameters from disturbance data and thus simulate the flywheel-imbalance-caused harmonic disturbances. However, due to the limitations of the empirical model in representing the large amplifications caused by the flywheel's internal flexibility at some speeds, in [22] the model was further developed by integrating it with a RWA mathematical model that accounted for the flywheel imbalance and internal resonances. In practice, however, WAs are installed on spacecraft structures, meaning that their dynamic behavior becomes coupled with that of the spacecraft (i.e., disturbances from the WA excite the spacecraft, which in turn excites the WA itself, and so forth, producing a dynamic coupling effect between the two bodies). For this reason, they cannot be treated in isolation and in $[22,23]$ a detailed method based on a combination of experimental data and analytical equations was developed to account for coupling effects between the RWA and its supporting structure. The structure performance model was developed with coupled RWA disturbances considered, and several experiments were carried out to validate the model and also for comparison to the two modeling methods (grounded and coupled WA disturbances as inputs). The results show that the coupled RWA-structure disturbance model when applied to the spacecraft structure finite element model as input gives a more accurate prediction than applying the grounded disturbance. However, in [23] the development of the mathematical model of the RWA (cantilevered flywheel with rigid bearing in this case) is not included, and input disturbance data are empirical (from RWA vibration tests). This method was then applied to assess the optical performance of the Space Interferometry Mission in [24,25].

In this paper, a mathematical model of a WA designed by Satellite Services Limited in collaboration with the University of Southampton is developed and its dynamics are studied in detail to provide a model that, in the future, can be integrated with a complete satellite model. This WA is presented in Figs. 1c and 1d. The WA is composed of a cantilevered flywheel supported by a soft-suspension system that constrains both rotations and translations. The suspension system also connects the motor/shaft and flywheel to the WA base. This is substantially different from the traditional arrangement, which has the shaft supported by bearings positioned at some distance along the shaft, and where the bearings are modeled as sets of springs and dashpots acting along orthogonal axes. The traditional model is widely used for general rotating systems, and its study has been well consolidated and can be found in many papers and textbooks [26-28], being a few examples. In our case the softsuspension system is instead connected at a single location, restrains translations and rotations, and can be designed as a passive control system to minimize the high-frequency disturbances induced by the

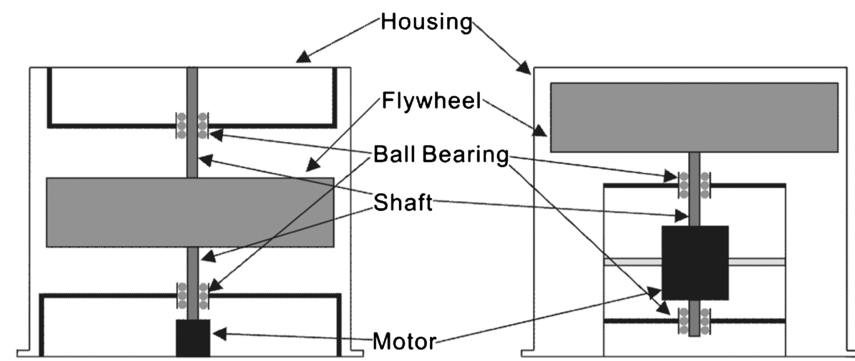

a) Symmetrical WA b) Cantilevered WA with rigid support

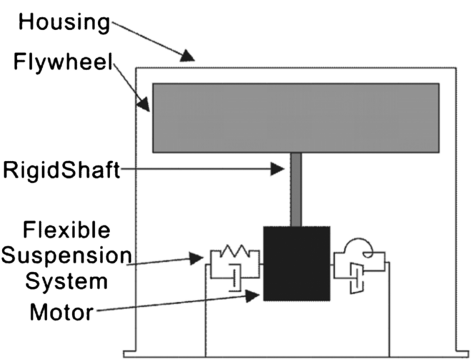

c) Cantilevered WA with soft suspension

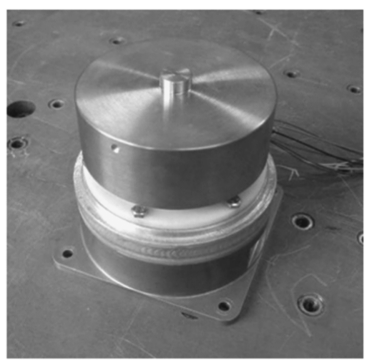

d) Cantilevered WA used in test

Fig. 1 Typical wheel configurations. 
WA. The system is modeled as a combination of linear and torsional springs and dashpots at a certain location offset from the c.m. of the rotor. In this paper, by using the Lagrangian approach (energy method), a mathematical model of this WA is developed. This model is then extended to include the dynamics of a seismic mass that is used to carry out microvibration tests (also described in this paper) to validate the mathematical models.

Coupled with the issue of modeling as described above, the accurate measurement of microvibrations induced by spacecraft units has also become an important subject. ESA has recently issued an Invitation to Tender (ITT) $\$$ to establish the best practice to be implemented in a facility for the characterization of sources of microvibrations.

To accurately measure WA-induced disturbances, many methods have been developed in the past, and the three most representative are reported in $[21,23,29]$. Generally speaking, dynamometric platforms (e.g., Kistler table) are the most used system for spacecraft equipment vibration tests. These are ideal for direct measurement of force and moment disturbances in six degrees of freedom (DOF) for grounded WAs with minimum detectable force in general down to $1 \mathrm{mN}$ [10]; however, they cannot be used for a coupled measurement, due to their size and weight. Other systems that can be used are based on the airfloating vibration detection system described in [29], which, in summary, uses a charge-coupled-device laser sensor to measure system displacement disturbance caused by the RWA, then converts this into force and momentum disturbances. The RWA-induced vibrations (grounded) were measured and analyzed later in [30] using this system. This type of system can accurately measure disturbances with frequency down to the subhertz region with a minimum detectable force of about $0.5 \mathrm{mN}$. However, the maximum frequency is only up to $20 \mathrm{~Hz}$, and only one lateral direction can be measured at one time. Finally, there are force-moment sensors such as those produced by JR3, Inc., which can be used for both grounded and coupled analysis [23]. However, these sensors usually contribute a significant mass to the test specimen. If the specimen is a small RWA, the requirement for high-accuracy measurements of low-level vibration may be jeopardized by this alteration in system mass (this issue only becomes severe for the coupled analysis).

Concerning the measurement system, in this paper, a relatively simple indirect method (seismic-mass microvibration measurement system) is proposed as an alternative to the systems described above. This method uses relatively common high-sensitivity accelerometers to measure the vibrations transmitted from the WA to a supporting seismic mass. In this paper, it is shown that the accuracies of the measurements that can be obtained are comparable with the other more sophisticated systems. Finally, the microvibrations emitted by a WA with a soft-suspension system are compared with those produced by a traditional rigid-suspension design.

\section{Mathematical Model}

Some typical WA configurations are schematically shown in Fig. 1. In the symmetrical configuration, Fig. 1a, the flywheel is fixed at midspan of the shaft, which is supported by bearings at each side of the flywheel. In Fig. 1b, a cantilever configuration is shown. It has the shaft on one side of the flywheel supported by a pairing of ball bearings at a certain distance apart from each other, with the motor in between. In these conventional designs, the components in the WAs are usually sized to provide enough stiffness to deliver a relatively high resonant frequency (normally above $100 \mathrm{~Hz}$ ) that meets the typical spacecraft requirements. However, if this requirement can be waived somehow (i.e., compared with the spacecraft, a RWA can be very small and its internal resonances at low frequencies may not affect the spacecraft global modes; thus, the minimum-frequency requirement can be waived and the wheel internal resonances can be considered as a local phenomena), the selection of the stiffness can be

\footnotetext{
\$"Advancement of Methodologies to Measure Micro Vibrations on Spacecraft Units," ESA ITT Ref. TEC-TCP/2009.37/MW/ AO 1-6201/09/ NL/CO, issued 23 June 2009.
}

made in order to minimize the mechanical disturbances produced by the WA.

In this paper, the WA studied has a cantilevered flywheel supported by a soft-suspension system, shown in Figs. 1c and 1d, which provides both rotational and translational support. The softsuspension system can be designed as a passive system that filters the vibrations produced by the motor (e.g., its bearings), thus minimizing the mechanical disturbances emitted by the WA when spinning. The WA is designed to have a speed range beyond $10,000 \mathrm{rpm}$, making it suitable for use as either a RWA or MWA.

In this section, the mathematical models of the disturbances emitted by the WA flywheel are developed, and they can be used for trading off the various parameters that characterize the WA design in order to minimize the disturbances and/or coupled with a model of the spacecraft to predict the vibrations at sensitive locations.

The WA's dynamic behavior is modeled using generalized equations of motion (EOM) through Lagrange's equations (energy approach):

$$
\frac{\mathrm{d}}{\mathrm{d} t}\left(\frac{\partial L}{\partial \dot{\xi}_{n}}\right)-\frac{\partial L}{\partial \xi_{n}}=Q_{i}
$$

where $\xi_{n}$ is the $n$th generalized coordinate, $Q_{i}$ is external work done, and $L$ is known as the Lagrangian and defined as the difference between total kinetic energy $T$ and total potential energy $U$ of the system:

$$
L\left(\xi_{1} \cdots \xi_{n}, \dot{\xi}_{1} \cdots \dot{\xi}_{n}, t\right)=T-U
$$

where $t$ is time, and the dot denotes the first derivative with respect to the time.

\section{A. Grounded WA}

Generally speaking, flywheel imbalance cannot be totally avoided, as there will always be some tolerances or imperfections due to manufacture. The development of the complete mathematical model (either a grounded WA or that coupled with a seismic mass) includes three cases: balanced flywheel, statically imbalanced flywheel and dynamically imbalanced flywheel. The imbalanced WA can be considered as the simplified model shown in Fig. 2:

From Fig. 2 , the inertial (fixed) frame $\left(X_{w}, Y_{w}\right.$, and $\left.Z_{w}\right)$ and body (rotating) frame $\left(x_{w}, y_{w}\right.$, and $\left.z_{w}\right)$ coincide at the c.m. of the flywheel $O$ with $z_{w}$ axis as the shaft-pointing direction. The corresponding

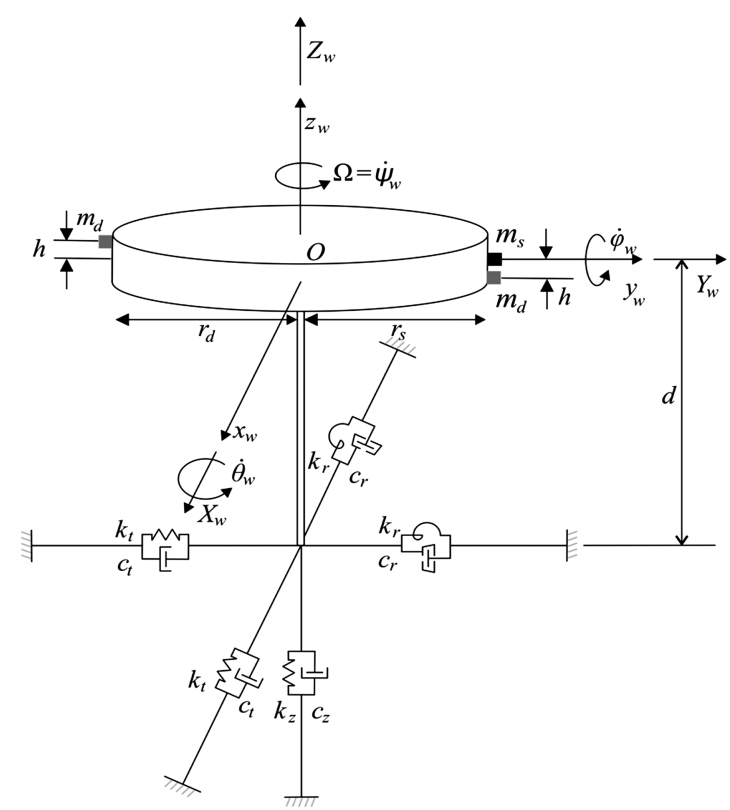

Fig. 2 Model of grounded cantilevered flywheel supported by a softsuspension system. 
rotations around each body axis are $\theta_{w}, \varphi_{w}$, and $\psi_{w}$, respectively (note that the flywheel spins at a constant speed: thus, $\dot{\psi}_{w}=\Omega$ ). The flywheel is modeled as a rigid thin disk with mass $M_{w}$, connected by a rigid massless shaft of length $d$ to a flexible axisymmetric support. The support is modeled as a pairing of linear and rotational springs and dashpots in the two lateral directions, respectively, in the rotation plane and in the shaft-pointing direction with a pairing of a linear spring and dashpot. Because of the axisymmetry of the flexible support, the linear spring stiffness $k_{t}$ is the same in the $x_{w} z_{w}$ and $y_{w} z_{w}$ planes, and the same applies to the torsional spring stiffness $k_{r}$ and dashpot damping coefficients $c_{t}$ and $c_{r}$. In the shaft-pointing direction, the linear spring stiffness and dashpot damping coefficient are $k_{z}$ and $c_{z}$, respectively.

For the statically imbalanced case, the imbalance can be modeled as a small point mass $m_{s}$ placed at radius $r_{s}$ on the flywheel. The dynamic imbalance is modeled using two identical small point masses $m_{d}$ placed $180^{\circ}$ apart at a radius distance $r_{d}$ and an axial distance $2 h$.

The inertia tensor of the idealized flywheel in the body frame is taken as

$$
\boldsymbol{I}_{w}=\left[\begin{array}{ccc}
I_{r} & 0 & 0 \\
0 & I_{r} & 0 \\
0 & 0 & I_{z}
\end{array}\right]
$$

where $I_{r}$ and $I_{z}$ are the moments of inertia about the transverse $\left(x_{w}\right.$ and $\left.y_{w}\right)$ axes and rotation $\left(z_{w}\right)$ axis, respectively. Euler transformations are used to write the angular velocity of the balanced wheel in the body frame as

$$
\boldsymbol{\omega}_{w}=\left\{\begin{array}{c}
\dot{\theta}_{w} \cos \psi_{w}+\dot{\varphi}_{w} \cos \theta_{w} \sin \psi_{w} \\
-\dot{\theta}_{w} \sin \psi_{w}+\dot{\varphi}_{w} \cos \theta_{w} \cos \psi_{w} \\
-\dot{\varphi}_{w} \sin \theta_{w}+\Omega
\end{array}\right\}
$$

The total kinetic energy of the flywheel is the sum of translational and rotational kinetic energy; thus,

$$
\begin{aligned}
T_{w} & =\frac{1}{2}\left[M_{w}\left(\dot{x}_{w}^{2}+\dot{y}_{w}^{2}+\dot{z}_{w}^{2}\right)+\left(\dot{\theta}_{w}^{2}+\dot{\varphi}_{w}^{2} \cos ^{2} \theta_{w}\right) I_{r}\right. \\
& \left.+\left(\dot{\varphi}_{w}^{2} \sin ^{2} \theta_{w}+\Omega^{2}-2 \Omega \dot{\varphi}_{w} \sin \theta_{w}\right) I_{z}\right]
\end{aligned}
$$

Similarly, the total potential energy of the nominal flywheel is the sum of lateral translational, rotational and axial potential energy. By assuming small displacements, the potential energy of the wheel can be expressed as

$$
\begin{aligned}
U_{w} & \approx \frac{1}{2}\left(k_{t} x_{w}^{2}-2 k_{t} \varphi_{w} d x_{w}+k_{r} \varphi_{w}^{2}+k_{t} d^{2} \varphi_{w}^{2}+k_{t} y_{w}^{2}+2 k_{t} d \theta_{w} y_{w}\right. \\
& \left.+k_{r} \theta_{w}^{2}+k_{t} d^{2} \theta_{w}^{2}+k_{z} z_{w}^{2}\right)
\end{aligned}
$$

For the static imbalanced case, the total displacement vector of the static imbalancing mass can be obtained as

$$
\boldsymbol{U}_{m s}=\left\{\begin{array}{c}
\left(-\cos \varphi_{w} \sin \Omega t+\sin \varphi_{w} \sin \theta_{w} \cos \Omega t\right) r_{s}+x_{w} \\
\left(\cos \theta_{w} \cos \Omega t\right) r_{s}+y_{w} \\
\left(\sin \varphi_{w} \sin \Omega t+\cos \varphi_{w} \sin \theta_{w} \cos \Omega t\right) r_{s}+z_{w}
\end{array}\right\}
$$

And the kinetic energy of the imbalancing mass $T_{m s}$ can be obtained as

$$
\begin{aligned}
T_{m s} & =\frac{1}{2} m_{s}\left\{\dot{x}_{w}^{2}+\dot{y}_{w}^{2}+\dot{z}_{w}^{2}+r_{s}^{2}\left[\dot{\varphi}_{w}^{2}\left(1-\cos ^{2} \Omega t \cos ^{2} \theta_{w}\right)\right.\right. \\
& \left.+\dot{\theta}_{w}^{2} \cos ^{2} \Omega t+\Omega^{2}\right]-2 r_{s} \dot{y}_{w}\left(\dot{\theta}_{w} \cos \Omega t \sin \theta_{w}\right. \\
& \left.+\Omega \cos \theta_{w} \sin \Omega t\right)+2 r_{s}^{2} \dot{\varphi}_{w}\left(-\Omega \sin \theta_{w}\right. \\
& \left.+\dot{\theta}_{w} \cos \Omega t \cos \theta_{w} \sin \Omega t\right)+2 r_{s} \dot{x}_{w}\left[\dot{\theta}_{w} \cos \Omega t \cos \theta_{w} \sin \varphi_{w}\right. \\
& -\Omega\left(\cos \Omega t \cos \varphi_{w}+\sin \Omega t \sin \theta_{w} \sin \varphi_{w}\right) \\
& \left.+\dot{\varphi}_{w}\left(\cos \Omega t \cos \varphi_{w} \sin \theta_{w}+\sin \Omega t \sin \varphi_{w}\right)\right] \\
& +r_{s} \dot{z}_{w}\left(-2 \Omega \cos \varphi_{w} \sin \Omega t \sin \theta_{w}+2 \Omega \cos \Omega t \sin \varphi_{w}\right. \\
& +2 \dot{\theta}_{w} \cos \Omega t \cos \theta_{w} \cos \varphi_{w}+2 \dot{\varphi}_{w} \cos \varphi_{w} \sin \Omega t \\
& \left.\left.-2 \dot{\varphi}_{w} \cos \Omega t \sin \theta_{w} \sin \varphi_{w}\right)\right\}
\end{aligned}
$$

Assuming small displacements, that the mass imbalance $m_{s}$ is much smaller than the flywheel's mass $M_{w}$, and that the wheel spin speed $\Omega$ is much larger than the rotational perturbation velocity about each axis, the kinetic energy of the statically imbalanced flywheel $T_{w+m s}$ can finally be obtained as

$$
\begin{aligned}
& T_{w+m s} \approx \frac{1}{2}\left[M_{w}\left(\dot{x}_{w}^{2}+\dot{y}_{w}^{2}+\dot{z}_{w}^{2}\right)+\dot{\theta}_{w}^{2} I_{r}+\dot{\varphi}_{w}^{2} I_{r}+\Omega^{2} I_{z}\right. \\
& \left.\quad-2 m_{s} r_{s} \dot{y}_{w} \Omega \sin \Omega t-2 m_{s} r_{s} \dot{x}_{w} \Omega \cos \Omega t-2 \dot{\varphi}_{w} \Omega \theta_{w} I_{z}\right]
\end{aligned}
$$

For the dynamic imbalanced case, to derive the kinetic energy of the two added masses, the total displacement vectors of each dynamic mass imbalance on the flywheel are first written down as

$$
\begin{aligned}
& \boldsymbol{U}_{m d 1} \\
& =\left[\begin{array}{c}
\left(-\cos \varphi_{w} \sin \Omega t+\sin \varphi_{w} \sin \theta_{w} \cos \Omega t\right) r_{d}-h \sin \varphi_{w} \cos \theta_{w}+x_{w} \\
\left(\cos \theta_{w} \cos \Omega t\right) r_{d}+h \sin \theta_{w}+y_{w} \\
\left(\sin \varphi_{w} \sin \Omega t+\cos \varphi_{w} \sin \theta_{w} \cos \Omega t\right) r_{d}-h \cos \varphi_{w} \cos \theta_{w}+z_{w}
\end{array}\right]
\end{aligned}
$$

$$
\begin{aligned}
& \boldsymbol{U}_{m d 2} \\
& =\left[\begin{array}{c}
-\left(-\cos \varphi_{w} \sin \Omega t+\sin \varphi_{w} \sin \theta_{w} \cos \Omega t\right) r_{d}+h \sin \varphi_{w} \cos \theta_{w}+x_{w} \\
-\left(\cos \theta_{w} \cos \Omega t\right) r_{d}-h \sin \theta_{w}+y_{w} \\
-\left(\sin \varphi_{w} \sin \Omega t+\cos \varphi_{w} \sin \theta_{w} \cos \Omega t\right) r_{d}+h \cos \varphi_{w} \cos \theta_{w}+z_{w}
\end{array}\right]
\end{aligned}
$$

where Eqs. (10) and (11) are the displacement vectors for the dynamic mass imbalance on the right-hand side and left-hand side, respectively, about the shaft axis in Fig. 2. The total kinetic energy of two imbalancing masses $T_{m d}$ can then be written as

$$
\begin{aligned}
T_{m d} & =m_{d}\left\{\dot{x}_{w}^{2}+\dot{y}_{w}^{2}+\dot{z}_{w}^{2}+r_{d}^{2} \Omega^{2}+\dot{\varphi}_{w}^{2}\left[h^{2} \cos ^{2} \theta_{w}\right.\right. \\
& \left.+r_{d}^{2}\left(1-\cos ^{2} \Omega t \cos ^{2} \theta_{w}\right)-2 r_{d} h \sin \theta_{w} \cos \theta_{w} \cos \Omega t\right] \\
& +\dot{\theta}_{w}^{2}\left(r_{d}^{2} \cos ^{2} \Omega t+h^{2}\right)-2 r_{d} \dot{\theta}_{w} \sin \Omega t[h \Omega \\
& \left.-\dot{\varphi}_{w}\left(r_{d} \cos \theta_{w} \cos \Omega t+h \sin \theta_{w}\right)\right]-2 r_{d} \dot{\varphi}_{w} \Omega\left(r_{d} \sin \theta_{w}\right. \\
& \left.\left.-h \cos \Omega t \cos \theta_{w}\right)\right\}
\end{aligned}
$$

Therefore, the total kinetic energy $T_{w+m s+m d}$ of the complete imbalanced flywheel can be obtained by summing Eqs. (5), (ㅁ) , and (12). Using the above-mentioned assumptions for the statically imbalanced case gives

$$
\begin{aligned}
& T_{w+m s+m d} \approx M_{w}\left(\dot{x}_{w}^{2}+\dot{y}_{w}^{2}+\dot{z}_{w}^{2}\right)+\dot{\theta}_{w}^{2} I_{r}+\dot{\varphi}_{w}^{2} I_{r}+\Omega^{2} I_{z} \\
& \quad-2 m_{s} r_{s} \dot{y}_{w} \Omega \sin \Omega t-2 m_{s} r_{s} \dot{x}_{w} \Omega \cos \Omega t-2 \dot{\varphi}_{w} \Omega \theta_{w} I_{z} \\
& \quad-4 r_{d} m_{d} h \dot{\theta}_{w} \Omega \sin \Omega t-4 r_{d} \dot{\varphi}_{w} \Omega\left(m_{d} r_{d} \theta_{w}-m_{d} h \cos \Omega t\right)
\end{aligned}
$$

Finally, the external work done can be obtained by applying virtual forces and moments at the c.m. of the flywheel, and, by using Eqs. (ㅁ) 
and (13), it is possible to write the Lagrangian, thus having all the terms present in Eq. (1). Using this equation, the resulting EOM can be written as

$$
\begin{aligned}
& {\left[\begin{array}{ccccc}
M_{w} & 0 & 0 & 0 & 0 \\
0 & I_{r} & 0 & 0 & 0 \\
0 & 0 & M_{w} & 0 & 0 \\
0 & 0 & 0 & I_{r} & 0 \\
0 & 0 & 0 & 0 & M_{w}
\end{array}\right]\left\{\begin{array}{c}
\ddot{x}_{w} \\
\ddot{\varphi}_{w} \\
\ddot{y}_{w} \\
\ddot{\theta}_{w} \\
\ddot{z}_{w}
\end{array}\right\}} \\
& +\left[\begin{array}{ccccc}
c_{t} & -c_{t} d & 0 & 0 & 0 \\
-c_{t} d & c_{r}+c_{t} d^{2} & 0 & -\Omega I_{z} & 0 \\
0 & 0 & c_{t} & c_{t} d & 0 \\
0 & \Omega I_{z} & c_{t} d & c_{r}+c_{t} d^{2} & 0 \\
0 & 0 & 0 & 0 & c_{z}
\end{array}\right]\left\{\begin{array}{c}
\dot{x}_{w} \\
\dot{\varphi}_{w} \\
\dot{y}_{w} \\
\dot{\theta}_{w} \\
\dot{z}_{w}
\end{array}\right\} \\
& +\left[\begin{array}{ccccc}
k_{t} & -k_{t} d & 0 & 0 & 0 \\
-k_{t} d & k_{r}+k_{t} d^{2} & 0 & 0 & 0 \\
0 & 0 & k_{t} & k_{t} d & 0 \\
0 & 0 & k_{t} d & k_{r}+k_{t} d^{2} & 0 \\
0 & 0 & 0 & 0 & k_{z}
\end{array}\right]\left\{\begin{array}{c}
x_{w} \\
\varphi_{w} \\
y_{w} \\
\theta_{w} \\
z_{w}
\end{array}\right\} \\
& =\left\{\begin{array}{c}
-m_{s} r_{s} \Omega^{2} \sin \Omega t \\
2 m_{d} r_{d} h \Omega^{2} \sin \Omega t \\
m_{s} r_{s} \Omega^{2} \cos \Omega t \\
2 m_{d} r_{d} h \Omega^{2} \cos \Omega t \\
0
\end{array}\right\}
\end{aligned}
$$

From Eq. (14), it is evident that the lateral translational and rotational motions in the two planes $x_{w} z_{w}$ and $y_{w} z_{w}$ are coupled, as neither the stiffness matrix nor the damping matrix is diagonal. Meanwhile, although for a cantilevered flywheel the two lateral motions are coupled, under reasonable assumptions (small displacement, small mass imbalance, and large flywheel spin speed), the static imbalance force does not affect the flywheel rotational (rocking) motion. The only result is an offset of the c.m. of the flywheel from its spin axis [although this does not appear in Eq. (14), due to small-mass imbalance assumption], and there is no external torque on the flywheel. Similarly, adding dynamic mass imbalances only produces a moment about the c.m. of the flywheel, with no effect on the lateral translational motion.

Equation (14) is in linearized form and includes the assumption that the mass imbalance does not change the flywheel mass and inertia properties captured in the mass matrix in Eq. (14). This is a reasonable assumption in practice, since flywheels are balanced quite well during manufacture, and the residual imbalance is relatively small compared with the flywheel. However, the mass imbalance cannot be neglected as a source of excitation [right-hand side in Eq. (14)], as the amplitudes of both force and moment disturbance are proportional to flywheel spin speed squared. For example, a typical MWA can spin at up to $10,000 \mathrm{rpm}$; as a result, despite the low mass imbalance, large loads can still be created, due to the high velocity. With the small-mass imbalance assumption, there is theoretically no excitation along the shaft-pointing direction.

\section{B. WA Coupled with Seismic Mass}

The quantification of the WA-induced disturbance is one of the most significant steps to assess the suitability of a design. In this paper, a relatively simple method is used to measure the disturbances emitted by the WA, and its implementation is shown in Fig. 3. In essence, this method consists of calculating the forces and moments produced by the WA by measuring the accelerations of a seismic mass that supports it.

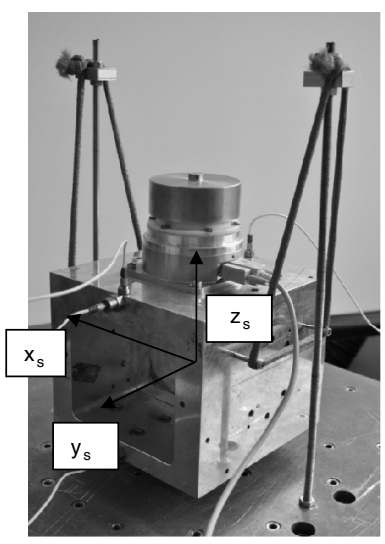

a)

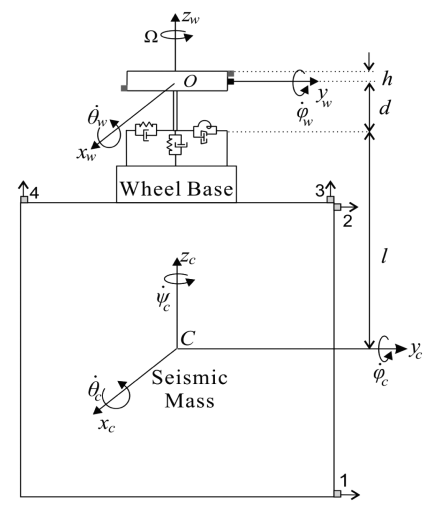

b)
Fig. 3 Microvibration measurement system: a) test setup, b) simplified model.

The whole system (WA and seismic mass, which also includes the wheel base) is suspended using elastic cords supported by benchfixed aluminum poles each side of the seismic mass, thus simulating a free-free condition. The elastic cords were chosen such that the system resonant frequencies were less than $1 \mathrm{~Hz}$ (verified with a tap test) and not in the frequency range of interest in this paper. The seismic mass has coordinates $x_{c} y_{c} z_{c}$ with origin $C$ at its c.m.; $\theta_{c}, \varphi_{c}$, and $\psi_{c}$ are the corresponding rotations around each axis. Note that in the shaft-pointing direction, the kinetic energy of the wheel is much larger than that of the seismic mass, and thus the perturbation of the seismic mass about this axis, $\psi_{c}$ is ignored. $M_{c}$ is the mass of the seismic mass; $I_{c_{x x}}$ and $I_{c_{y y}}$ are moments of inertia of the seismic mass about the $x_{c}$ and $y_{c}$ axes, respectively. The vertical distance from the soft-suspension-system/wheel-base interface to the c.m. of the seismic mass is $l$.

The seismic mass can be considered as a rigid body connected with a flywheel (another rigid body) by the soft-suspension system. With the small-displacement assumption, the kinetic energy of the seismic mass $T_{c}$ is obtained as

$$
T_{c} \approx \frac{1}{2}\left[M_{c}\left(\dot{x}_{c}^{2}+\dot{y}_{c}^{2}+\dot{z}_{c}^{2}\right)+I_{c_{x x}} \dot{\theta}_{c}^{2}+I_{c_{y y}} \dot{\varphi}_{c}^{2}\right]
$$

The potential energy $U_{s}$ of the system can be obtained (using the small-displacement assumption) through the relative motion at the soft-suspension-system/flywheel-base interface:

$$
\begin{aligned}
& U_{s}=\frac{1}{2}\left[k_{z}\left(z_{w}-z_{c}\right)^{2}+k_{r}\left(\theta_{w}-\theta_{c}\right)^{2}+k_{t}\left[\left(y_{w}-y_{c}\right)+\left(d \theta_{w}\right.\right.\right. \\
& \left.\left.\left.+l \theta_{c}\right)\right]^{2}+k_{r}\left(\varphi_{w}-\varphi_{c}\right)^{2}+k_{t}\left[\left(x_{w}-x_{c}\right)-\left(d \varphi_{w}+l \varphi_{c}\right)\right]^{2}\right]
\end{aligned}
$$

Using Eq. (1) for the undamped system, the fully linearized EOM of the system with respect to each of the $10 \mathrm{DOF}$ (the axial rotation of flywheel and seismic mass are neglected) are obtained. They are written in matrix form as

$$
\boldsymbol{M}_{s} \ddot{\boldsymbol{q}}_{s}+\boldsymbol{G}_{s} \dot{\boldsymbol{q}}_{s}+\boldsymbol{K}_{s} \boldsymbol{q}_{s}=\boldsymbol{F}_{s}
$$

where all matrices are as follows:

$$
\boldsymbol{G}_{s}=\left[\begin{array}{cccccccccc}
0 & 0 & 0 & 0 & 0 & 0 & 0 & 0 & 0 & 0 \\
0 & 0 & 0 & 0 & 0 & 0 & 0 & 0 & 0 & 0 \\
0 & 0 & 0 & 0 & 0 & 0 & 0 & 0 & 0 & 0 \\
0 & 0 & 0 & 0 & \Omega I_{z} & 0 & 0 & 0 & 0 & 0 \\
0 & 0 & 0 & -\Omega I_{z} & 0 & 0 & 0 & 0 & 0 & 0 \\
0 & 0 & 0 & 0 & 0 & 0 & 0 & 0 & 0 & 0 \\
0 & 0 & 0 & 0 & 0 & 0 & 0 & 0 & 0 & 0 \\
0 & 0 & 0 & 0 & 0 & 0 & 0 & 0 & 0 & 0 \\
0 & 0 & 0 & 0 & 0 & 0 & 0 & 0 & 0 & 0 \\
0 & 0 & 0 & 0 & 0 & 0 & 0 & 0 & 0 & 0
\end{array}\right]
$$




$$
\boldsymbol{M}_{s}=\left[\begin{array}{cccccccccc}
M_{w} & 0 & 0 & 0 & 0 & 0 & 0 & 0 & 0 & 0 \\
0 & M_{w} & 0 & 0 & 0 & 0 & 0 & 0 & 0 & 0 \\
0 & 0 & M_{w} & 0 & 0 & 0 & 0 & 0 & 0 & 0 \\
0 & 0 & 0 & I_{r} & 0 & 0 & 0 & 0 & 0 & 0 \\
0 & 0 & 0 & 0 & I_{r} & 0 & 0 & 0 & 0 & 0 \\
0 & 0 & 0 & 0 & 0 & M_{c} & 0 & 0 & 0 & 0 \\
0 & 0 & 0 & 0 & 0 & 0 & M_{c} & 0 & 0 & 0 \\
0 & 0 & 0 & 0 & 0 & 0 & 0 & M_{c} & 0 & 0 \\
0 & 0 & 0 & 0 & 0 & 0 & 0 & 0 & I_{c_{x x}} & 0 \\
0 & 0 & 0 & 0 & 0 & 0 & 0 & 0 & 0 & I_{c_{y y}}
\end{array}\right]
$$

$$
\begin{gathered}
\omega^{4}-\Omega \frac{I_{z}}{I_{r}} \omega^{3}-\left(\frac{k_{t}}{M_{w}}+\frac{k_{r}+k_{t} d^{2}}{I_{r}}\right) \omega^{2}+\Omega \frac{k_{t} I_{z}}{M_{w} I_{r}} \omega+\frac{k_{t} k_{r}}{M_{w} I_{r}}=0 \\
\omega_{z}=\sqrt{\frac{k_{z}}{M_{w}}}
\end{gathered}
$$

where $\omega$ refers to the four flexural natural frequencies and $\omega_{z}$ is the axial one. For the case when the WA is coupled with the seismic mass, Eq. (17) can be solved numerically. The frequencies $\omega$

$$
\boldsymbol{K}_{s}=\left[\begin{array}{cccccccccc}
k_{t} & 0 & 0 & 0 & -d k_{t} & -k_{t} & 0 & 0 & 0 & -l k_{t} \\
0 & k_{t} & 0 & d k_{t} & 0 & 0 & -k_{t} & 0 & l k_{t} & 0 \\
0 & 0 & k_{z} & 0 & 0 & 0 & 0 & -k_{z} & 0 & 0 \\
0 & d k_{t} & 0 & d^{2} k_{t}+k_{r} & 0 & 0 & -d k_{t} & 0 & l d k_{t}-k_{r} & 0 \\
-d k_{t} & 0 & 0 & 0 & d^{2} k_{t}+k_{r} & d k_{t} & 0 & 0 & 0 & d l k_{t}-k_{r} \\
-k_{t} & 0 & 0 & 0 & d k_{t} & k_{t} & 0 & 0 & 0 & l k_{t} \\
0 & -k_{t} & 0 & -d k_{t} & 0 & 0 & k_{t} & 0 & -l k_{t} & 0 \\
0 & 0 & -k_{z} & 0 & 0 & 0 & 0 & k_{z} & 0 & 0 \\
0 & l k_{t} & 0 & l d k_{t}-k_{r} & 0 & 0 & -l k_{t} & 0 & l^{2}+k_{r} & 0 \\
-l k_{t} & 0 & 0 & 0 & l d k_{t}-k_{r} & l k_{t} & 0 & 0 & 0 & l^{2} k_{t}+k_{r}
\end{array}\right]
$$

$$
\boldsymbol{q}_{s}=\left\{\begin{array}{c}
x_{w} \\
y_{w} \\
z_{w} \\
\theta_{w} \\
\varphi_{w} \\
x_{c} \\
y_{c} \\
z_{c} \\
\theta_{c} \\
\varphi_{c}
\end{array}\right\} \quad \boldsymbol{F}_{s}=\left\{\begin{array}{c}
-m_{s} r_{s} \Omega^{2} \sin \Omega t \\
m_{s} r_{s} \Omega^{2} \cos \Omega t \\
0 \\
2 m_{d} r_{d} h \Omega^{2} \cos \Omega t \\
2 m_{d} r_{d} h \Omega^{2} \sin \Omega t \\
0 \\
0 \\
0 \\
0 \\
0
\end{array}\right\}
$$

Note that if the seismic mass is fixed (i.e., grounded WA), i.e., the $\mathrm{DOF}$ of the seismic mass are ignored, then Eq. (17) reduces to Eq. (14).

\section{Natural Frequencies}

If the WA is directly grounded (i.e., with the seismic mass blocked), Eq. (14) can be written in complex coordinates and then solved using the usual homogeneous equation. The biquadratic equations for flexural motion and the independent axial motion of the undamped system can thus be obtained as obtained from Eqs. (17-19) are plotted individually as functions of the flywheel spin speed $\Omega$ in a Campbell diagram, as shown in Figs. $4 \mathrm{a}$ and $4 \mathrm{~b}$. Vibration tests were carried out for both configurations (details shown in Sec. III), and the frequencies where the fundamental harmonic and five modes appear were extracted from the test data and then superimposed in Figs. $4 \mathrm{a}$ and $4 \mathrm{~b}$ in order to verify the theoretical values.

From Figs. $4 \mathrm{a}$ and $4 \mathrm{~b}$, among the four real roots for both cases, two are positive. $\bar{\omega}_{3}$ and $\omega_{4}$ occur in the forward direction (forward whirling), $\omega_{1}$ and $\omega_{2}$ occur in the backward direction (backward whirling). Since the axial motion is uncoupled from lateral motion, $\omega_{z}$ is solved independently and resulted in a horizontal line on the diagram. Also, it is notable in the test results that $\omega_{2}$ backward whirling is not seen in any test, and $\omega_{1}$ backward whirling is hardly seen, but forward whirling is easily identified in both cases.

The predictions of the five theoretical solutions at standstill are listed in Table 1. Owing to axisymmetry, there are two identical pairs; which this is also clear from Fig. 4 at $0 \mathrm{rpm}$. To identify experimental values of the WA at standstill, tests were carried out and these results are also listed in Table 1.

Generally speaking, test results match very well with all theoretical curves; the largest error is approximately $2 \mathrm{~Hz}$, occurring

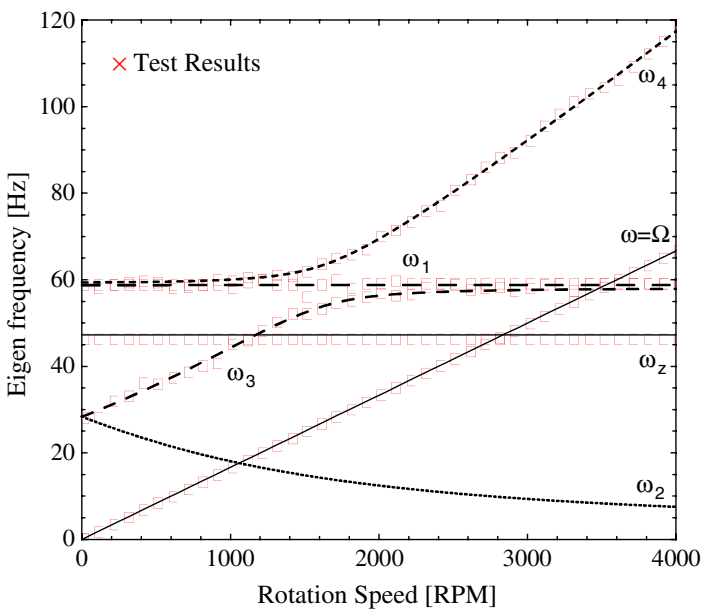

b)

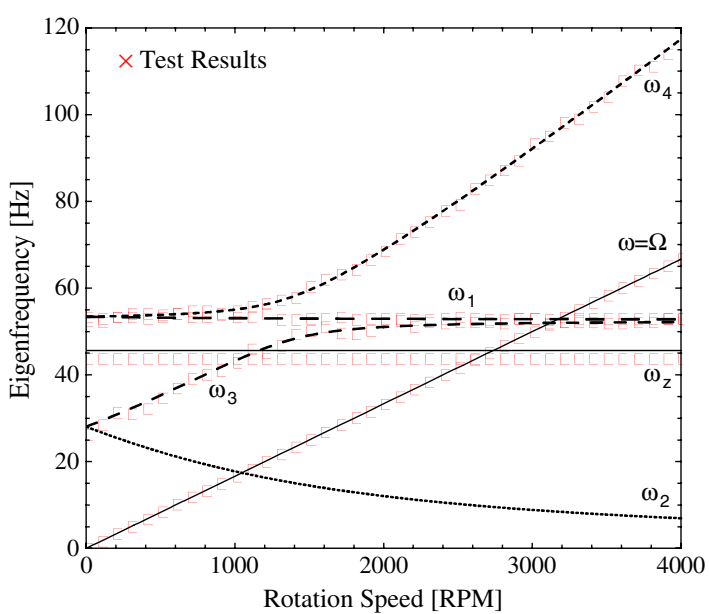


Table 1 Standstill whirling modes and natural frequencies for the grounded and coupled wheels

\begin{tabular}{clcccc}
\hline \hline & & \multicolumn{2}{c}{ Grounded wheel, Hz } & \multicolumn{2}{c}{ Coupled wheel, Hz } \\
\cline { 3 - 6 } Solutions & Whirling mode & Theoretical & Experimental & Theoretical & Experimental \\
\hline$\omega_{1}$ & Lateral backward & 53 & 52 & 59 & 58 \\
$\omega_{2}$ & Lateral backward & 28 & 26 & 28 & 28 \\
$\omega_{3}$ & Lateral forward & 28 & 26 & 28 & 28 \\
$\omega_{4}$ & Lateral forward & 53 & 52 & 59 & 58 \\
$\omega_{z}$ & Axial & 46 & 44 & 47 & 46 \\
\hline \hline
\end{tabular}

Table 2 Theoretical and experimental synchronous critical speeds of flexible WAs (grounded and coupled)

\begin{tabular}{cccc}
\hline \hline \multicolumn{4}{c}{ Synchronous critical speeds, rpm } \\
Theoretical & Testing \\
\hline$x$ & Grounded flexible WA & \\
3120 & $y$ & $x$ & $y$ \\
& 3120 & 3300 & 3100 \\
3480 & Coupled flexible $W A$ & \\
\hline \hline
\end{tabular}

for the axial motion (the relative error is $8 \%$ ). Some discrepancies are due to the small-mass assumption used in the mathematical models. For example, using Eq. (19) for the grounded case and considering the mass imbalance of $0 . \overline{2} \mathrm{~g}$ at the outmost edge of the flywheel, the experimentally measured axial natural frequency matches exactly the theoretical one of $28 \mathrm{~Hz}$.

Meanwhile, as expected for a cantilevered flywheel, from Figs. $4 \mathrm{a}$ and $4 \mathrm{~b}$ the two forward whirls $\left(\omega_{3}\right.$ and $\left.\omega_{4}\right)$ and the lateral translational and rocking modes are coupled with each other. In this case, it is impossible to separate the lateral translational and lateral rocking whirling on each of the two curves. As speed increases, one whirling type transforms to the other. For example, for the grounded case Fig. $4 \mathrm{a}, \omega_{3}$ curve appears to be lateral rocking from 0 to $1300 \mathrm{rpm}$ (the sloped region of the graph), then tends toward lateral translational motion as speed increases to infinity (the horizontal part). On the same diagram, the $\omega_{4}$ curve shows the opposite behavior. The coupled case has a similar pattern for all natural frequencies, with the difference that the mixed lateral translational modes shift upward by $5 \mathrm{~Hz}$, whereas the rest do not change significantly. This will increase the synchronous critical speed slightly (see Sec. IV, Table 2). Also, the mode turning point for the two forward modes shifts to $1700 \mathrm{rpm}$. This means that by considering the WA coupled with seismic mass, the motion of the flywheel becomes more translational in the given speed range. Because of this mixed-mode feature, it is difficult to distinguish amplitudes belonging to the different modes at around $50 \mathrm{~Hz}$ in the test results.

In conclusion, at this point, it is possible to say that two mathematical models given in Eqs. (14) and (17) can be used to describe the undamped and free behavior of the system with appropriate accuracy.

\section{Microvibration Measurement}

A Kistler table was used to verify the theoretical results for the case of the WA directly grounded. Since this is the most common method to carry out microvibration measurements, these tests will be described briefly. For the WA coupled with the seismic mass shown in Fig. 3, a more in-depth discussion is provided. All tests were carried out in a quiet environment, in a noise-isolated lab basement at night; all electrical devices such as air conditioning, redundant computers, and computer fan, etc., were shut down and discounted; the data-acquisition system (the computer) was isolated from the ground on a trolley; and test setups (Kistler table and coupled measurement system) were put on a shaker bench that was lifted by air cushions to isolate any noise transmitted from the ground. No audible acoustic sources (speech sound) were present during measurement.

\section{A. Grounded Microvibration Tests}

Disturbance forces induced by grounded WA were directly measured using a six-axis Kistler force/torque table (9253B12). The test setup is shown in Fig. $\underline{5}$.

An aluminum fixture was used as an interface between the table and WA. The disturbances induced from the flywheel were directly transmitted to the base of the fixture, which was mounted in the center of the table. The four sensors at each corner of the table measured the forces, which were then combined using Eq. (20) for output:

$$
\left\{\begin{array}{l}
F_{x}=F_{x 1+2}+F_{x 3+4} \\
F_{y}=F_{y 1+4}+F_{y 2+3} \\
F_{z}=F_{z 1}+F_{z 2}+F_{z 3}+F_{z 4}
\end{array}\right.
$$

The output charge signals are converted into force signals through a three-channel charge amplifier (model Kistler 5019B) and are then acquired by a data-acquisition board (National Instruments PCI4472). This set up was used to obtain the experimental resonances, which are then superimposed with the theoretical curves in Fig. 4a. However, note that tests in a grounded configuration will not completely reflect the dynamic behavior of the WA when it is attached to the spacecraft.

\section{B. Coupled Microvibration Test}

The system used to analyze the behavior of the WA on the seismic mass has been presented in Fig. 3. Four accelerometers (Endevco

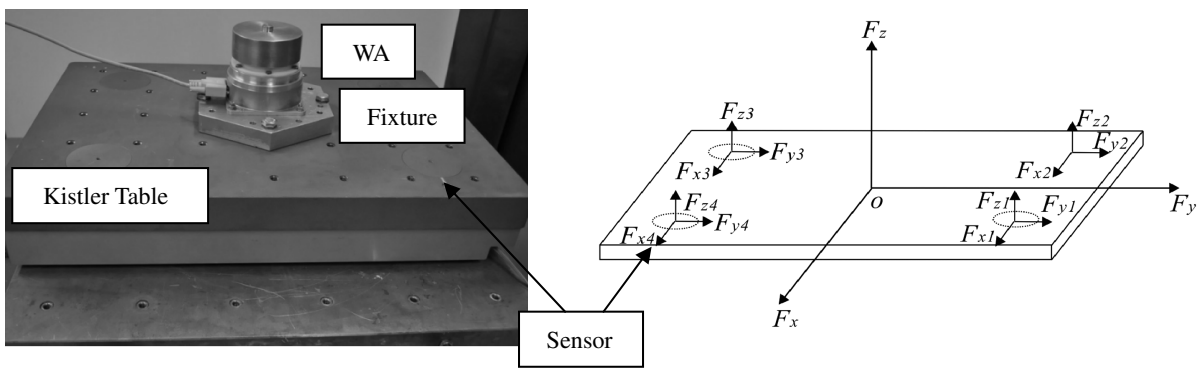

Fig. 5 Grounded wheel tests: a) test setup and b) Kistler table model. 
752A13) were attached to the seismic mass at the locations shown in the figure to measure the acceleration signals.

Using the coordinate system $x_{s} y_{s} z_{s}$ with origin $O^{\prime}$ at the c.m. of the system, the corresponding system rotation speeds are $\omega_{s x}, \omega_{s y}$, and $\omega_{s z}$, respectively. Accelerometers 1 and 2 are used to measure acceleration signals in the lateral direction with distances $d_{1}$ and $d_{2}$, respectively, from the $y_{s}$ axis. Accelerometers 3 and 4 are used to measure acceleration signals in the shaft-pointing direction, $s$ is the distance of point mass $m_{e}$ on the flywheel from the $y_{s}$ axis, and $d_{3}$ is the half-width of the seismic mass.

In the following subsections, the accelerations measured at the four accelerometer locations are first compared with the corresponding theoretical values. Next, a generalized inertia matrix is derived to transform acceleration signals at the four locations into forces and moments at the WA-base/seismic-mass interface. Finally, the performance of the seismic-mass microvibration measurement system is investigated.

\section{Acceleration Verification}

To verify the acceleration signals acquired by the four accelerometers with the theoretical ones, the rigid-WA model is used. Since the system does not have any resonances below $2000 \mathrm{rpm}$ for both flexible and rigid WAs, there are no dynamic amplifications due to resonances if the WA is operated at low speeds and the whole system (WA supported by the seismic mass) behaves like a rigid body. In practice, this is achieved by replacing the soft-suspension system with a rigid-suspension system. From Fig. 6, the four lateral EOM under the action of imbalance forces $F_{s x}$ and $F_{s y}$ and moments $M_{s x}$ and $M_{s y}$ at $O^{\prime}$ in the two symmetric planes $x_{s} z_{s}$ and $y_{s} z_{s}$ can be written in the standard form:

$$
\begin{gathered}
\left\{\begin{array}{l}
F_{s x}=M_{s} \ddot{x}_{s} \\
F_{s y}=M_{s} \ddot{y}_{s}
\end{array}\right. \\
\left\{\begin{array}{l}
M_{s x}=\dot{\omega}_{s x} I_{s_{x x}}+\omega_{s z} \omega_{s y}\left(I_{s_{z z}}-I_{s_{y y}}\right) \\
M_{s y}=\dot{\omega}_{s y} I_{s_{y y}}+\omega_{s z} \omega_{s x}\left(I_{s_{x x}}-I_{s_{z z}}\right)
\end{array}\right.
\end{gathered}
$$

where $M_{s}$ and $I_{s_{x x}}, I_{s_{y y}}$, and $I_{s_{z z}}$ are the mass and the moments of inertia about three axes of the system, respectively.

From conservation of angular momentum, the following relationship can be obtained for the WA and seismic-mass system:

$$
\omega_{s z} I_{s z}=I_{z} \Omega+I_{c_{z z}} \omega_{b z}
$$

In the shaft-pointing direction, at constant speed, the flywheel spin speed $\Omega$ is much larger than the perturbation speed of the seismic mass $\omega_{b z}$, and so Eq. (23) can be simplified as

$$
\omega_{s z} \approx \frac{I_{z}}{I_{s_{z z}}} \Omega
$$

By inserting Eq. (24) into Eq. (22), and expressing explicitly the moment produced by the rotation of the point mass $m_{e}$ (see Fig. 6), the following alternative form of the equations is obtained:

$$
\left\{\begin{array}{l}
-m_{e} \Omega^{2} r s \sin (\Omega t)=\dot{\omega}_{s x} I_{s_{x x}}+\frac{I_{z}}{I_{s z z}} \Omega \omega_{s y}\left(I_{s_{z z}}-I_{s_{y y}}\right) \\
m_{e} \Omega^{2} r s \sin \left(\Omega t+\frac{\pi}{2}\right)=\dot{\omega}_{s z} I_{s_{y y}}+\frac{I_{z}}{I_{s z z}} \Omega \omega_{s x}\left(I_{s_{x x}}-I_{s_{z z}}\right)
\end{array}\right.
$$

where $r$ is the radius of the flywheel. Equation $(25)$ is then used to calculate numerically $\omega_{s x}$ as a function of the time $t$.

The acceleration at the four accelerometer locations can then be derived from the angular velocity and rotation:

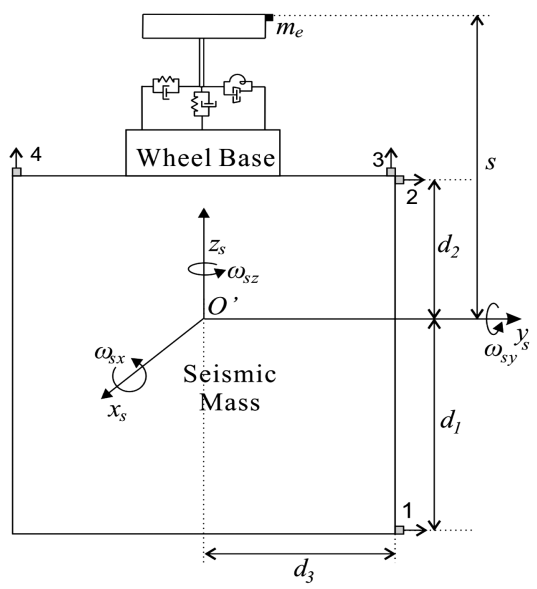

Fig. 6 Simplified model of microvibration measurement system (global system).

$$
\left\{\begin{array}{l}
a_{1}=\frac{m_{s} \Omega^{2} r \sin (\Omega t)}{M_{s}}+\dot{\omega}_{s x} d_{1}-\omega_{s x}^{2} d_{3} \\
a_{2}=\frac{m_{s} \Omega^{2} r \sin (\Omega t)}{M_{s}}-\dot{\omega}_{s x} d_{2}-\omega_{s x}^{2} d_{3} \\
a_{3}=\dot{\omega}_{s x} d_{3}-\omega_{s x}^{2} d_{2} \\
a_{4}=-\dot{\omega}_{s x} d_{3}-\omega_{s x}^{2} d_{2}
\end{array}\right.
$$

Note that for the $a_{1}$ and $a_{2}$ expressions in Eq. (26), the first term is the translational acceleration, the second component comes from the tangential acceleration, and the third comes from the normal component, due to the rotational velocity. For $a_{3}$ and $a_{4}$, since, in theory, there is no excitation along the shaft longitudinal direction, there are only the second and third terms, due to the rotation of the seismic mass. In Eq. (26) the second-order seismic-mass perturbation speed terms are ignored, due to their small amplitudes compared with wheel spin speed and perturbation accelerations.

By inserting $\omega_{s x}$ calculated from Eq. (25), the acceleration of each accelerometer location (at each rotational speed) can be derived. These theoretical values are plotted in Fig. 7.

Several tests were carried out with a point mass $m_{e}$ of $1 \mathrm{~g}$ attached to the flywheel spinning at speeds from 0 to $2000 \mathrm{rpm}$ in steps of $100 \mathrm{rpm}$. The average values of the fundamental harmonic amplitude of 10 repeated tests for the four accelerometers were taken and plotted together with theoretical curves in Fig. 7.

From Fig. 7, the experimental results correlate well with the corresponding theoretical ones; the largest error is less than $2 \%$, which occurred for accelerometer 2 at $300 \mathrm{rpm}$. Note that although the difference between predictions and measured values is increasing

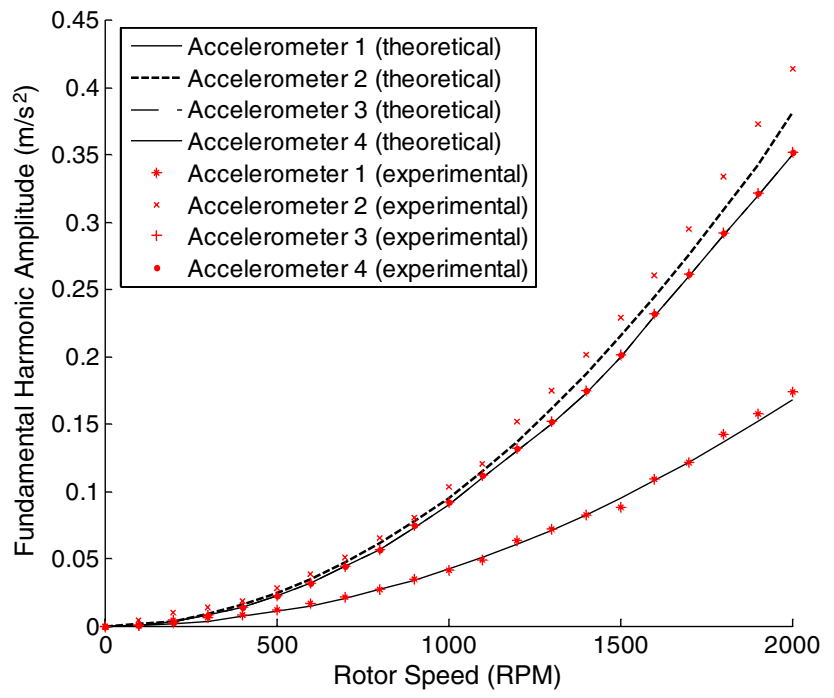

Fig. 7 Acceleration verification for four accelerometers. 


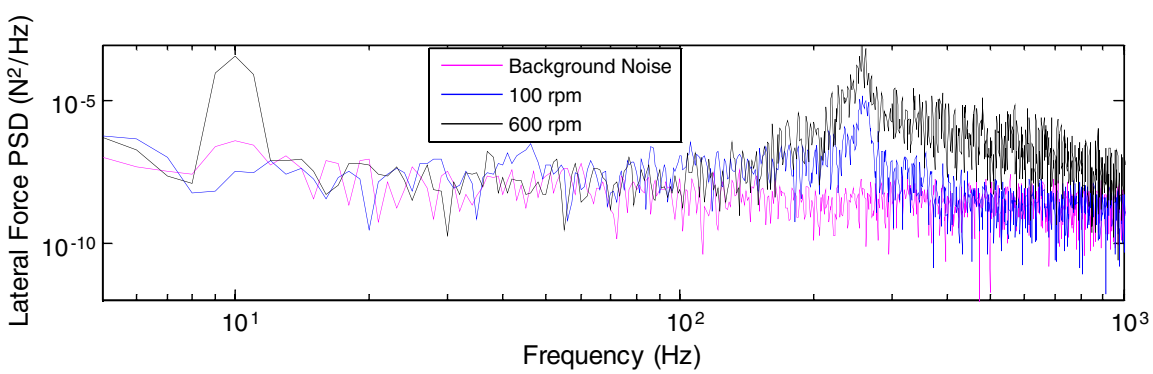

Fig. 8 Lateral force background noise of rigid system and disturbances at 100 and $600 \mathrm{rpm}$.

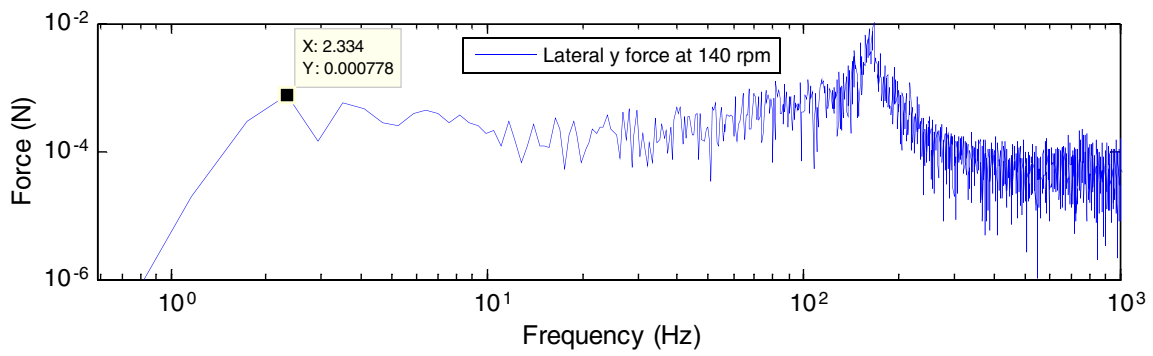

Fig. 9 Minimum detectable force in lateral direction of the rigid system (140 rpm).

the percentage error with respect to the measured value remains fairly constant. Accelerometers 3 and 4 have the same absolute amplitude, due to axisymmetry of the system and so in Fig. $\underline{7}$, the $a_{3}$ and $a_{4}$ results coincide both for their theoretical and experimental values. Meanwhile from Fig. 6, accelerometers 3 (and 4) are at almost the same horizontal level as accelerometer 2. Therefore, though they measure vibration signals in the two orthogonal directions, they give very close absolute amplitudes, as can be seen in Fig. 7. The good correlation between theoretical and experimental values for all accelerometers indicates that Eq. (26) can accurately describe accelerations at the four locations.

\section{Generalized Inertia Matrix}

The generalized inertia matrix is defined as the matrix that transforms linear and angular accelerations into forces and moments. Using Eqs. (21) and (26), the following equation is obtained:

$$
\left\{\begin{array}{l}
a_{1} \approx \frac{F_{s y}}{M_{s}}+\frac{M_{s x}}{I_{s_{x x}}} d_{1} \\
a_{2} \approx \frac{F_{s y}}{M_{s}}-\frac{M_{s x}}{I_{s_{x x}}} d_{2} \\
a_{3} \approx \frac{M_{s x}}{I_{s_{x x}}} d_{3} \\
a_{4} \approx-\frac{M_{s x}}{I_{s_{x x}}} d_{3}
\end{array}\right.
$$

From Eq. (27), the force $F_{s y}$ and moment $M_{s x}$ produced by the flywheel's imbalance at the c.m. of the system can be obtained. Thus, the lateral force $F_{i y}$ and moment $M_{i x}$ at the interface between the WA and the seismic mass are derived by transforming $F_{s y}$ and $M_{s x}$ as follows:

$$
\left[\begin{array}{c}
F_{i y} \\
M_{i x}
\end{array}\right]=\frac{1}{d_{1}+d_{2}}\left[\begin{array}{cc}
M_{s} d_{2} & M_{s} d_{1} \\
M_{s} d_{2}^{2}+I_{s_{x x}} & M_{s} d_{2} d_{1}-I_{s_{x x}}
\end{array}\right]\left[\begin{array}{l}
a_{1} \\
a_{2}
\end{array}\right]
$$

The generalized inertia matrix in Eq. (28) is then used to transform the acceleration signals at positions 1 and 2 into force and moment signals at the WA-base/seismic-mass interface in the $y_{s} z_{s}$ plane. Since the WA is axisymmetric, the absolute values of $F_{i x}$ and $M_{i y}$ in the $x_{s} z_{s}$ plane are the same as in the $y_{s} z_{s}$ plane. In the shaft-pointing direction, the disturbance moment at the WA-base/support interface can be obtained from the $a_{3}$ and $a_{4}$ expressions in either Eq. (27) or Eq. (28). Note that due to axisymmetry of the system, the absolute values detected by accelerometers 3 and 4 are the same all the time, but in opposite directions. Also $M_{i x}$ can be obtained from accelerometers 3 and 4 to verify the value obtained from accelerometers 1 and 2 using Eq. (28).
3. Seismic-Mass Microvibration Measurement System Performance

In the time domain at steady state, the lateral vibration signals acquired by accelerometers 1 and 2 are transformed into force/ moment signals at the interface between the WA and seismic mass by Eq. (28). They are then processed to obtain the corresponding power spectral density and amplitude spectrum of the disturbances at each speed (including the background noise). All tests were carried out in a quiet environment, and the seismic-mass microvibration measurement system was placed on a shaker bench supported by air cushions in order to isolate any possible disturbance transmitted from the ground. The background noise on the force measurement is mainly the result of electrical noise and its level greatly depends on the accelerometer sensitivity. As an example, Fig. 8 compares lateral $\left(y_{s}\right.$ direction) force background noise and disturbance at 100 and $600 \mathrm{rpm}$. Root-mean-square (rms) values are 2.5, 12, and $91 \mathrm{mN}$, respectively.

From Fig. $\underline{8}$, the background noise level is mostly below the two disturbances produced when the flywheel is spinning (also in terms of rms values). Note that the level of the noise is relatively flat, whereas spinning the flywheel, besides the peak at the rotation speed, produces substantial noise above $250 \mathrm{~Hz}$, which corresponds to the resonant frequency of the WA with rigid suspension replaced with the

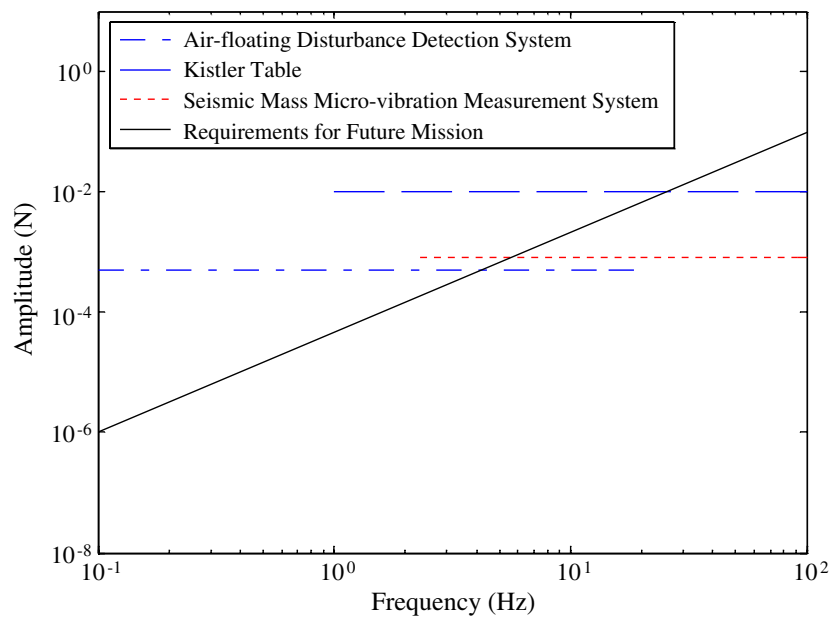

Fig. 10 Practical detectable disturbance level of available microvibration measurement method. 
soft-suspension system (this should simulate the behavior of a typical reaction wheel as that in Fig. 1b).

The minimum detectable force in the lateral direction was found by spinning the flywheel with a $0.2 \mathrm{~g}$ point mass attached on it, starting from $0 \mathrm{rpm}$ and increasing speed in $10 \mathrm{rpm}$ steps. With background noise considered (i.e., the practical performance), the first distinct peak at spin speed is found at $140 \mathrm{rpm}$ with amplitude $0.8 \mathrm{mN}$, as shown in Fig. 9 .

Figure 10 compares the microvibration measurement system performance with other available methods and also the requirements for future high-precision spacecraft design [7].

As seen from Fig. 10, the practical dynamic performance of the microvibration measurement system is between the air-floating detector (which is particularly good at measuring low-frequency disturbances; these are usually very difficult to detect, but have a maximum frequency around $20 \mathrm{~Hz}$ ) and typical a Kistler table (which usually has a wide detecting range, but with coarse resolution). Though the seismic-mass microvibration measurement system still does not satisfy the requirements for future spacecraft design, as a direct and accurate method, it can be used as an alternative solution.

\section{Soft-Suspension System}

To assess the soft-suspension system design in the WA, two types of tests were carried out. One was conducted with the soft-suspension system set as in service, i.e., flexible case (Fig. 1c); the other was run with the soft-suspension system replaced by a rigid-suspension system, corresponding to a conventional ball bearing design (e.g., Fig. 1b). All tests were carried out using the seismic-mass microvibration measurement system described before. The flywheel was spun from 0 to $7000 \mathrm{rpm}$ in $100 \mathrm{rpm}$ steps. The generalized inertia matrix in Eq. (28) is used to transform the acceleration signals acquired at the four locations shown in Fig. 6 into force and moment at the interface between the WA and the seismic mass. Provided that the accelerometers are positioned in the other plane of symmetry, the seismic-mass microvibration measurement system can measure simultaneously in both planes; however, the system was set up to measure in one plane here, and the WA was rotated by $90^{\circ}$ to measure disturbance induced in another lateral direction. The two sets of measurements were then combined to produce the force-tube plots described in the next section.

\section{A. Fundamental Harmonic Force Amplitude}

For each testing speed, the disturbance force amplitude of the fundamental harmonic caused by the flywheel imbalance was recorded in both planes and combined to plot a force orbit at each constant speed. The cumulative force orbits for all speeds can be presented as a tube. Force-orbit plots for the flexible- and rigid-WA cases are presented in following figures (Figs. 11-13).

From Fig. 11, it is clear that there is one resonance for the flexible case (in either lateral direction), but none for the rigid case, as, in fact, its first resonance frequency is beyond the range that was investigated. For the rigid case, the tube ends up with much larger amplitude for the same reason. To examine the behavior in detail, the flexible case is presented in the $x z$ and $y z$ planes, as shown in the following figure.

From Figs. $12 \mathrm{a}$ and $12 \mathrm{~b}$ in both directions, before approaching the first critical speed (resonance), the amplitude first increases steadily with a circular orbit up to $4 \mathrm{~N}$ at $3000 \mathrm{rpm}$. However, at resonance it is clear that both maximum amplitude and critical speed are different in the two planes. In the $x$ direction, the peak amplitude is about $4.5 \mathrm{~N}$ at $3600 \mathrm{rpm}$, but in the $y$ direction, it is $6.1 \mathrm{~N}$ at $3450 \mathrm{rpm}$. This nonsynchronous amplitude results in the orbit being highly elliptic in this region, but as the spin speed continues increasing, the orbit tends toward more or less a circle again, due to the self-centering effect of imbalance at high speed.

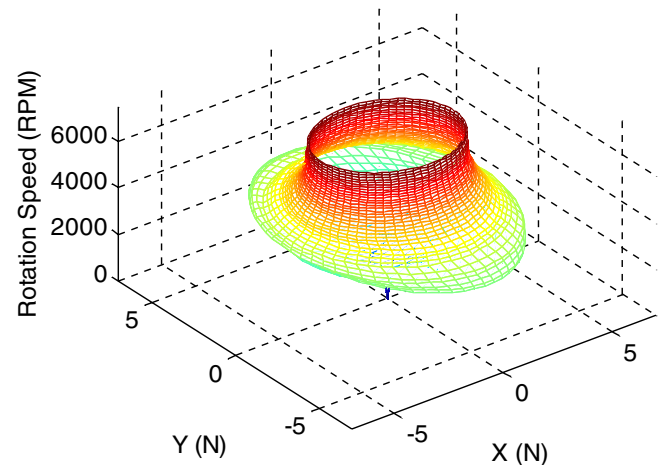

a)

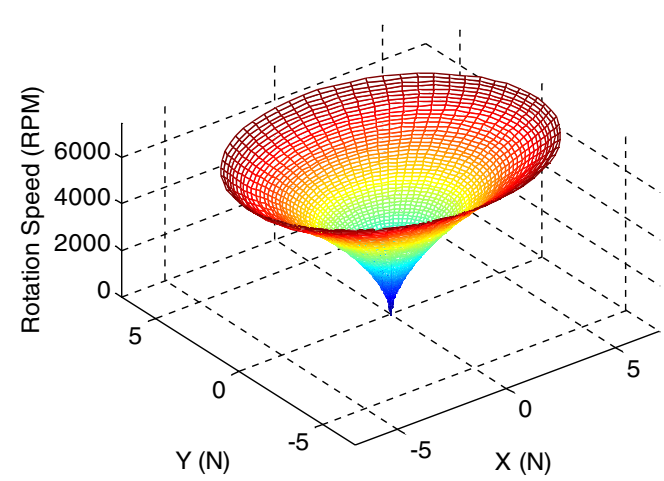

b)

Fig. 11 Force tube of a) flexible and b) rigid WA coupled with seismic mass.

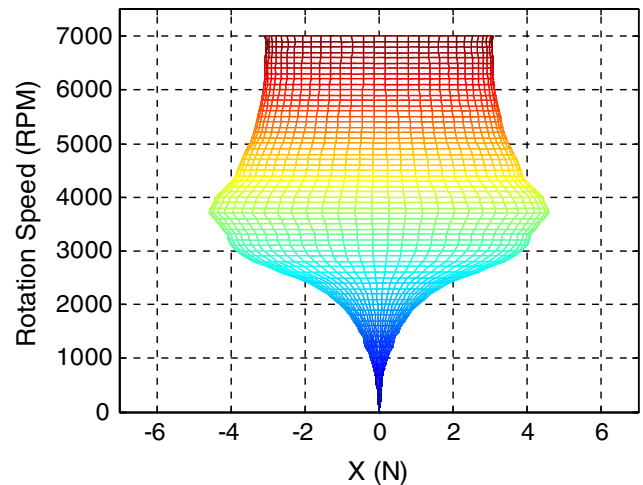

a)

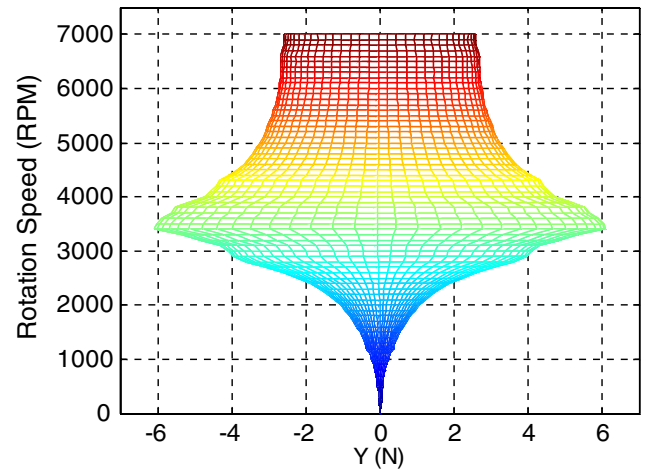

b)

Fig. 12 Imbalance amplitudes of flexible WA coupled with the seismic mass in a) $x z$ plane and b) $y z$ plane. 
Table 2 summarizes both theoretical and experimental synchronous critical speeds in the $x$ and $y$ directions for the flexible WA grounded and coupled with the seismic mass. Investigation of the critical speeds listed in Table $\underline{2}$ allows one to gain an insight into the elliptic orbit phenomena and the performance of the WA.

Since in theory, the WA is axisymmetric (as for both the flywheel and the soft-suspension system), the disturbance amplitude and critical speed in the $x$ and $y$ directions should be the same all the time even for the resonance. However, the test results in Table 2 show differences in the two directions. The critical speed of the WA on the seismic mass in the $y$ direction $(3450 \mathrm{rpm})$ is very close to the theoretical one (3480 rpm). Meanwhile, for the grounded case, the experimental critical speed in $y$ direction $(3100 \mathrm{rpm})$ is also very close to that of the theoretical one $(3120 \mathrm{rpm})$. Along the $x$ direction, the differences are significantly larger (about 120 and $180 \mathrm{rpm}$ ), and several tests were carried out to investigate the reason. It was found that the cables that connected the motor to the case, and also the external cable to connect the WA to the control unit (fixed on the ground) provided a further stiffness path along the $x$ direction.

Also from Table 2, the effect of mounting the WA on the seismic mass is clear. For experimental values in both directions, the difference is about 300 to $350 \mathrm{rpm}$ (around $5.5 \mathrm{~Hz}$ ), which is very close to the theoretical prediction of $360 \mathrm{rpm}$ shown in Table $\underline{2}$ and also Figs. $4 \mathrm{a}$ and $\underline{4 b}$.

For the rigid case, Fig. 13 shows the forces in the $x z$ and $y z$ planes. In this case, the amplitude increases steadily in the test-speed range, i.e., no resonance occurs, due to the high stiffness of the rigid bearing. At the maximum test speed of $7000 \mathrm{rpm}$, the imbalance amplitude reaches $7 \mathrm{~N}$ in the $x$ direction and $5.5 \mathrm{~N}$ in the $y$ direction.

\section{B. Soft-Suspension-System Performance}

To assess the soft-suspension-system performance, cascade plots are generated for both rigid and flexible cases. Figures $14 \mathrm{a}$ and $14 \mathrm{~b}$ present the force disturbance cascade plot in the lateral $y \overline{\text { direction for }}$ the flexible and rigid cases, respectively. Figure 15 presents those for the moments.

From Figs. $14 \mathrm{a}$ and $15 \mathrm{a}$, it is clear that a mixed translational mode occurs around $\overline{60} \mathrm{~Hz}$. $\overline{\text { Meanwhile, there are several superharmonics }}$ and one subharmonic appearing on the plot, due to the motor and its bearing disturbances, which are as expected, but no resonances over $60 \mathrm{~Hz}$. Note that other whirls (mixed rocking and backward) cannot be seen clearly on the cascade plots, due to large amplifications caused by flywheel imbalance across the entire test-speed range. Instead, they are identified individually from amplitude-requency plots at each spin speed, as shown in Fig. 16 (the flywheel rotating at $2400 \mathrm{rpm}$ for the two cases of cascade plots Figs. 14a and 14b), for example.

Compared with the rigid case, Figs. $14 \mathrm{~b}$ and $15 \mathrm{~b}$ show a large number of superharmonics appearing, $\overline{\text { due }}$ to the motor and its bearing disturbances, but in this case, since the bearing is rigid, the influences of these imperfections become more severe, resulting in more obvious superharmonics. Most importantly, the translational mode in this case appears around $280 \mathrm{~Hz}$ (in fact, a bugle between 200 and $400 \mathrm{~Hz}$ ), as shown in Fig. 16.

From Fig. 17, it is clear that at high-frequency band (between 100 to $700 \mathrm{~Hz}$ ), disturbances generated by the flexible WA are much less than those produced from the rigid WA, which produces significant disturbances at the high-frequency band between 200 and $450 \mathrm{~Hz}$. This validates those observed from Figs. 14 and 16 .

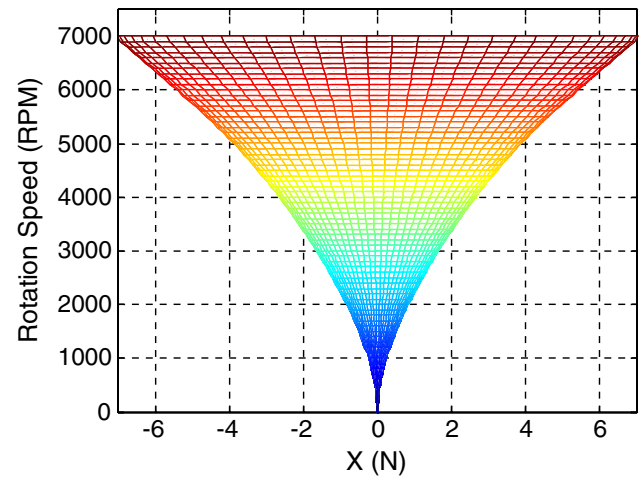

a)

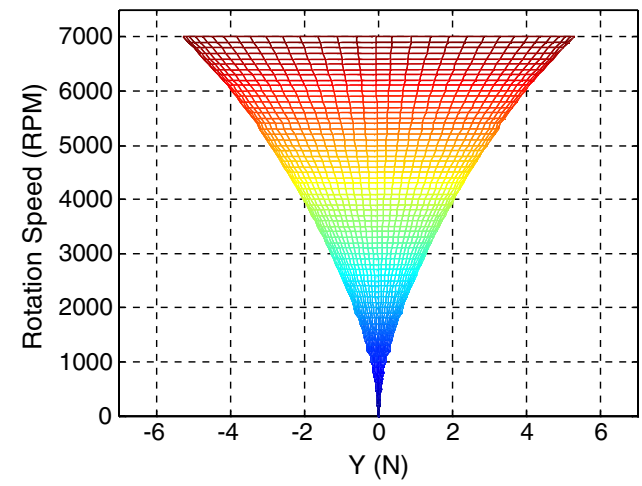

b)

Fig. 13 Imbalance amplitudes of rigid WA coupled with the seismic mass in a) $x z$ plane and b) $y z$ plane.
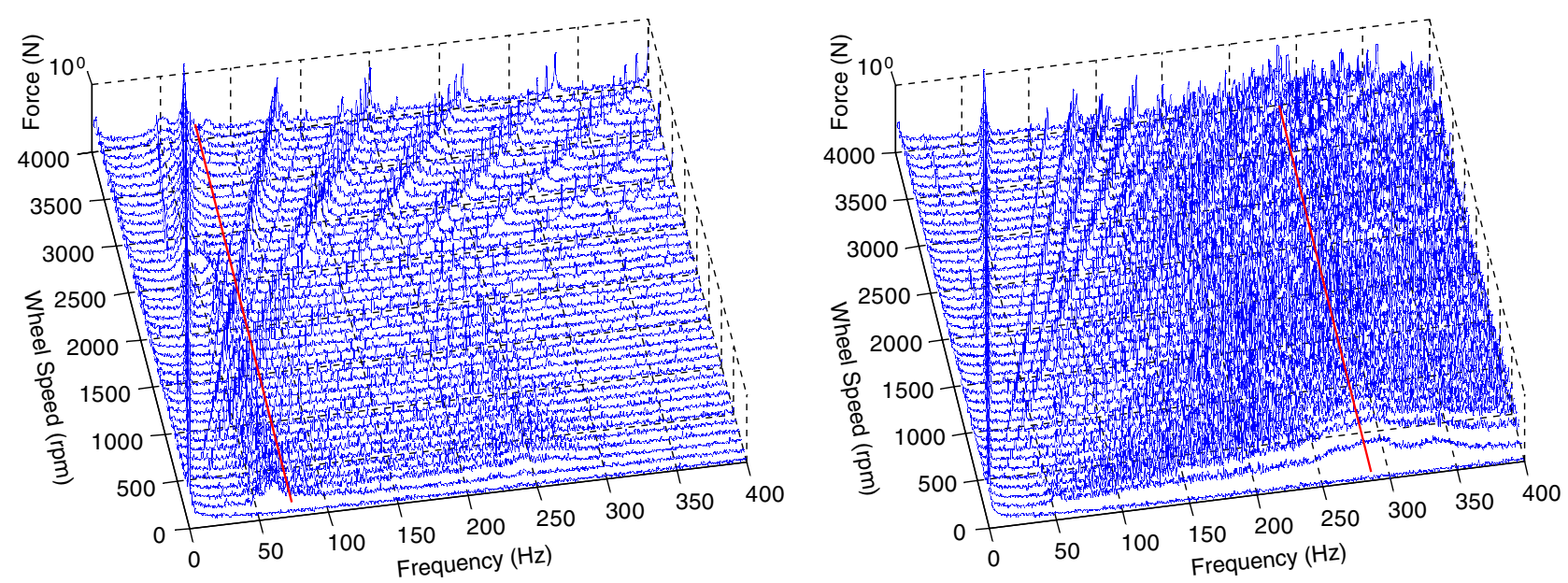

a)

b)

Fig. 14 Cascade plots of WA-induced force disturbance in $y$ axis of a) flexible and b) rigid case. 


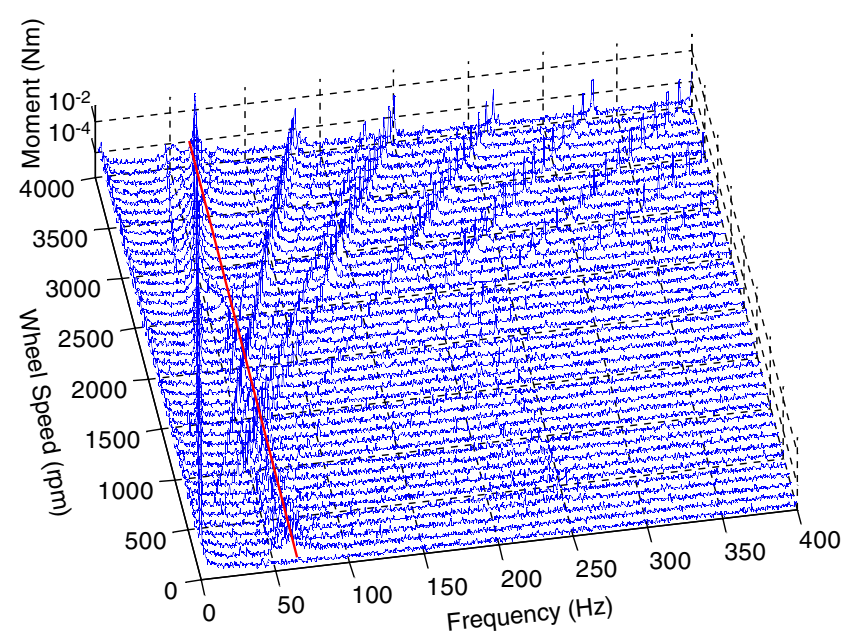

a)

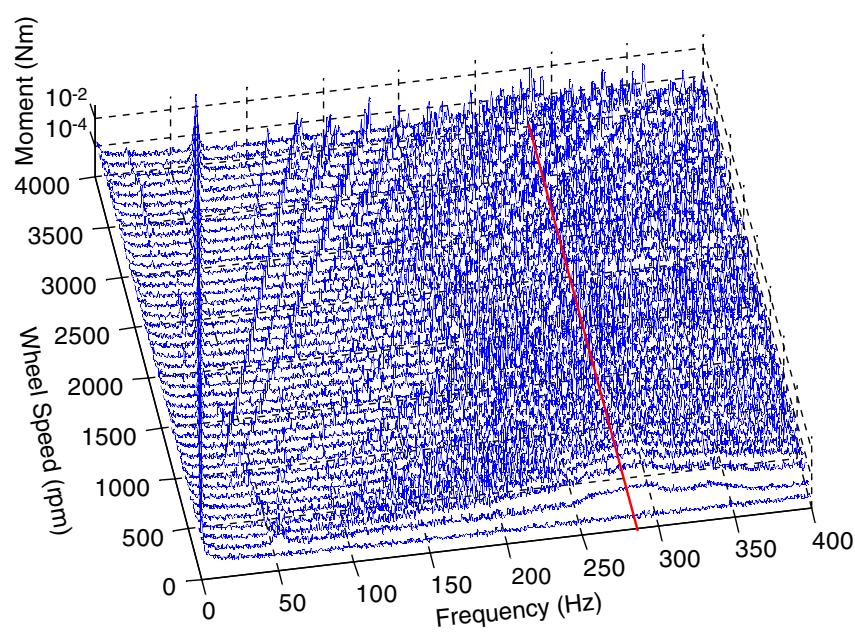

b)

Fig. 15 Cascade plots of WA-induced moment disturbance about $x$ axis a) flexible and b) rigid case.

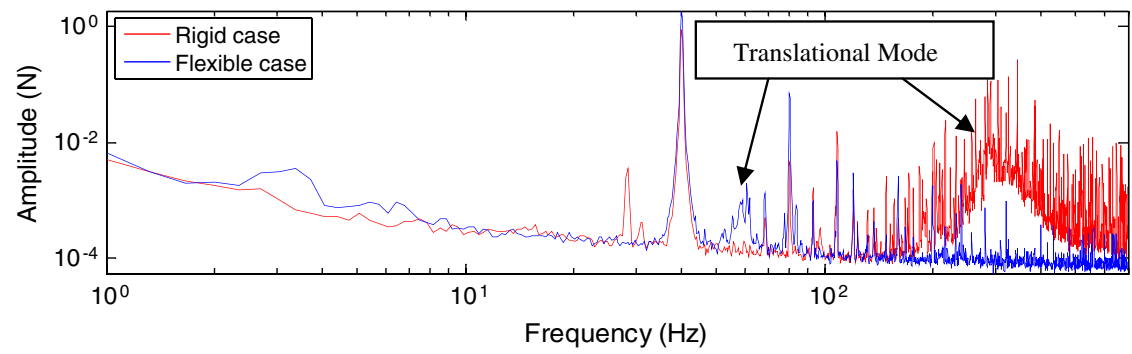

Fig. 16 Comparison of $y$ disturbance force for coupled flexible and rigid wheel at $2400 \mathrm{rpm}$.

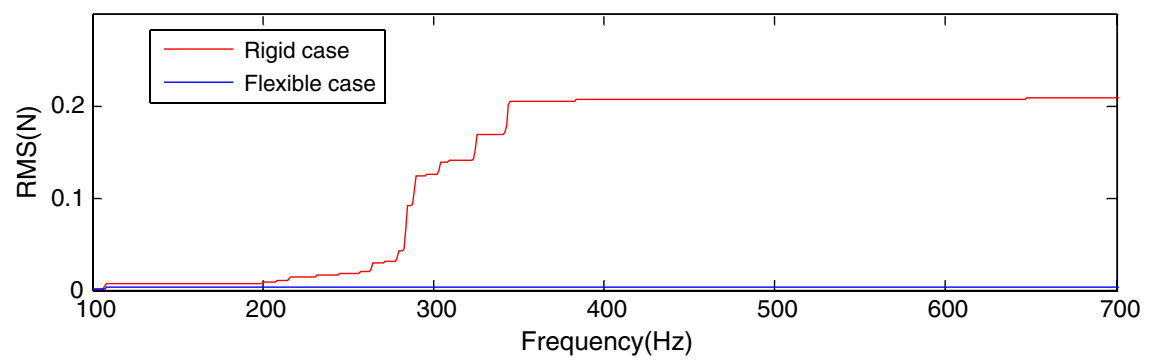

Fig. 17 Cumulative rms comparison of coupled flexible and rigid wheel at $2400 \mathrm{rpm}$ between 100 and $700 \mathrm{~Hz}$.

Traditionally, MWAs, which usually work at very high spin speed, produce high-frequency disturbances that are difficult to control and are likely to degrade the performances of sensitive equipments on spacecraft. However from the above discussion, it appears that the soft-suspension system can reduce such problems. For RWA applications, which usually work at much lower speed, the disturbances will be larger than those from a rigid design. However, these lowfrequency disturbances at specific frequencies may be easier to control than the higher-frequency broadband vibrations produced by a rigid WA.

\section{Conclusions}

In this paper, a mathematical model of flywheel-induced vibrations of a WA with a cantilever flywheel configuration supported by a soft-suspension system has been developed. The purpose of the model is to calculate the microvibrations emitted by the WA: i.e., reaction forces and moments at the interface between the WA and the ground or a supporting structure (in this work, a seismic mass). The paper also presents a simple indirect measurement system for the reactions at the WA mounting interface, based on monitoring the accelerations of a seismic mass onto which the WA is mounted. A generalized inertia matrix that allows the accelerations monitored during the experiment to be transformed into the interface loads is derived, and a comparison between the results of the mathematical model and experimental data shows a very good agreement. The resonant frequencies and amplitudes of the loads, produced by the static and dynamic imbalance of the flywheel during operation are correctly reproduced. The experimental setup could correctly measure WA-induced vibrations in lateral and axial direction simultaneously with a minimum detectable force below $1 \mathrm{mN}$ and minimum frequency below $2.5 \mathrm{~Hz}$. Finally, the performance of this WA in terms of microvibration emissions was compared with a traditional design (with a rigid suspension) through comparison of frequency spectra, and it was shown that this design produces significantly lower high-frequency vibrations.

\section{Acknowledgment}

The author would like to thank Satellite Services Limited for their support. 


\section{References}

[1] Bely, P. Y., Lupie, O. L., and Hershey, J. L., "Line-of-Sight Jitter of the Hubble Space Telescope," Proceedings of SPIE, Vol. 1945, 1993, pp. 55-61. doi: $10.1117 / 12.158800$

[2] Wacker, T., Weimer, L., and Eckert, K., "GOCE Platform MicroVibration Verification by Test and Analysis," Proceedings of the European Conference on Spacecraft Structures, Materials and Mechanical Testing, Noordwijk, The Netherlands, 2005.

[3] Takahara, O., Yoshida, N., Minesugi, K., Hashimoto, T., Ninomiya, K., Ichimoto, K., et al., "Microvibration Transmissibility Test of Solar-B," 24th International Symposium on Space Technology and Science, Miyazaki, Japan, 2004.

[4] Aglietti, G. S., Langley, R. S., and Gabriel, S. B., "Model Building and Verification for Active Control of Microvibrations with Probabilistic Assessment of the Effects of Uncertainties," Proceedings of the Institution of Mechanical Engineers Part C, Mechanical Engineering Science, Vol. 218, No. 4, 2004, pp. 389, 399.

[5] Laskin, R. A., and Martin, M. S., "Control/Structure System Design of a Spaceborne Optical Interferometer," Proceedings of the AAS/AIAA Astrodynamics Specialist Conference, AAS Paper 89-424, 1989.

[6] Davis, L. P., Wilson, J. F., Jewell, R. E., and Roden, J. J., "Hubble Space Telescope Reaction Wheel Assembly Vibration Isolation System," NASA Marshall Space Flight Center, Redstone Arsenal, AL, 1986.

[7] "An Evaluation of Reaction Wheel Emitted Vibrations for Large Space Telescope," NASA Marshall Space Flight Center, Rept. N76-18213, Redstone Arsenal, AL, Jan. 1976.

[8] Pendergast, K. J., and Schauwecker, C. J., "Use of a Passive Reaction Wheel Jitter Isolation System to Meet the Advanced $X$-Ray Astrophysics Facility Imaging Performance Requirements," Proceedings of SPIE, Vol. 3356, 1998, pp. 1078-1094. doi: $10.1117 / 12.324508$

[9] Narayan, S. S., Nair, P. S., and Ghosal, A., "Dynamic Interaction of Rotating Momentum Wheels with Spacecraft Element," Journal of Sound and Vibration, Vol. 315, Nos. 4-5, Sept. 2008, pp. 970-984. doi:10.1016/j.jsv.2008.02.020

[10] Hasha, M. D., "Reaction Wheel Mechanical Noise Variations," Space Telescope Program, Engineering Memo No. SSS 218, June 1998.

[11] Hyde, T. T., and Anderson, E. H., "Actuator with Built-In Viscous Damping for Isolation and Structural Control," AIAA Journal, Vol. 34, No. 1,1996 , pp. 129-135.

doi: $10.2514 / 3.13032$

[12] Oh, H., Izawa, K., and Taniwaki, S., "Development of VariableDamping Isolator Using Bio-Metal Fiber for Reaction Wheel Vibration Isolation," Smart Materials and Structures, Vol. 14, No. 5, 2005, pp. $928-933$. doi:10.1088/0964-1726/14/5/029

[13] Vaillon, L., and Philippe, C., "Passive and Active Microvibration Control for Very High Pointing Accuracy Space Systems," Smart Materials and Structures, Vol. 8, No. 6, 1999, pp. 719-728. doi:10.1088/0964-1726/8/6/302

[14] Fortescue, P., Stark, J., and Swind, G. (ed.), Spacecraft Systems Engineering, 3rd ed., Wiley Chichester, England, U.K., 2003, Chaps. 9.

[15] Kennedy, H. B., "A Gyro Momentum Exchange Device for Space Vehicle Attitude Control," AIAA Journal, Vol. 1, No. 5, 1963, pp. 1110 1118 .

doi: $10.2514 / 3.1732$

[16] Genta, G., Dynamics of Rotating Systems, Springer, New York, 2005,
Chaps. 2, 3.

[17] Muszyńska, A., Rotordynamics, Taylor \& Francis, Boca Raton, FL, 2005, Chaps. 3.

[18] Bialke, B., "A Compilation of Reaction Wheel Induced Spacecraft Disturbances," 20th Annual American Aeronautical Society Guidance and Control Conference, AAS Paper 97-038, 1997.

[19] Bialke, B., "High Fidelity Mathematical Modeling of Reaction Wheel Performance," 21st Annual American Aeronautical Society Guidance and Control Conference, AAS Paper 98-063, 1998.

[20] Stromswold, E., and Bialke, B., "Behavior of Reaction Wheels Near Zero Speed," Proceedings of the Annual AAS Guidance and Control Conference, Vol. 118, Breckenridge, CO, 2004, p. 361.

[21] Masterson, R. A., and Miller, D. W., "Development and Validation of Reaction Wheel Disturbance Models: Empirical Model," Journal of Sound and Vibration, Vol. 249, No. 3, Jan. 2002, pp. 575-598. doi:10.1006/isvi.2001.3868

[22] Masterson, R. A., and Miller, D. W., "Development of Empirical and Analytical Reaction Wheel Disturbance Models," Proceedings of the AIAA/ASME/AHS/ASC Structures, Structural Dynamics and Materials Conference, April 1999.

[23] Elias, L. M., and Miller, D. W., "A Coupled Disturbance Analysis Method Using Dynamic Mass Measurement Techniques," Proceedings of the AIAA/ASME/AHS/ASC Structures, Structural Dynamics and Materials Conference, April 2002.

[24] Elias, L. M., Dekens, F. G., Basdogan, I., Sievers, L. A., and Neville, T., "Methodology for Modeling the Mechanical Interaction Between a Reaction Wheel and a Flexible Structure," Proceedings of SPIE, Vol. 4852, 2003, pp. 541-555. doi: $10.1117 / 12.460875$

[25] Bas dogan, I., Elias, L. M., Dekens, F., and Sievers, L., "Predicting the Optical Performance of the Space Interferometry Mission Using a Modeling, Testing, and Validation Methodology," Journal of Vibration and Acoustics, Vol. 129, No. 2, 2007, pp. 148-157. doi:10.1115/1.2202152

[26] Carnegie, W., "The Application of Variational Method to Derive the Equations of Motion of Vibrating Cantilever Blading Under Rotation," Bulletin of Mechanical Engineering Education, Vol. 6, 1967, p. 29.

[27] Bavastri, C. A., da Silva Ferreira, E. M., de Espíndola, J. J., and Lopes, E. M. O., "Modeling of Dynamic Rotors with Flexible Bearings Due to the Use of Viscoelastic Materials," Journal of the Brazilian Society of Mechanical Sciences and Engineering, Vol. 30, No. 1, March 2008, pp. 22-29. doi: $10.1590 / \mathrm{S} 1678-58782008000100004$

[28] Adams, M. L., Jr., Rotating Machinery Vibration, from Analysis to Troubleshooting, Marcel Dekker, New York, 2000, Chaps. 2, pp. 4546.

[29] Taniwaki, S., and Ohkami, Y., "Experimental and Numerical Analysis of Reaction Wheel Disturbances," JSME International Journal, Series $C$, Vol. 46, No. 2, 2003, pp. 519-526. doi:10.1299/jsmec.46.519

[30] Taniwaki, S., Kudo, M., Sato, M., and Ohkami, Y., "Analysis of Retainer Induced Disturbances of Reaction Wheel," Journal of System Design and Dynamics, Vol. 1, No. 2, 2007, pp. 307-317. doi: $10.1299 /$ jsdd.1.307 